\title{
Characterization of senescence-associated protease activities involved in the efficient protein remobilization during leaf senescence of winter oilseed rape
}

\author{
Marine Poret $^{\mathrm{a}, \mathrm{b}, \mathrm{c}, *}$, Balakumaran Chandrasekar ${ }^{\mathrm{d}, \mathrm{e}}$, Renier A.L. van der Hoorn ${ }^{\mathrm{d}}$, \\ Jean-Christophe Avice ${ }^{\mathrm{a}, \mathrm{b}, \mathrm{c}, *}$ \\ a Université de Caen Normandie, F-14032 Caen, France \\ ${ }^{\mathrm{b}}$ UCBN, UMR INRA-UCBN 950 Ecophysiologie Végétale, Agronomie E' Nutritions N.C.S., F-14032 Caen, France \\ ' INRA, UMR INRA-UCBN 950 Ecophysiologie Végétale, Agronomie E Nutritions N.C.S., F-14032 Caen, France \\ d The Plant Chemetics Laboratory, Department of Plant Sciences, University of Oxford, South Parks Road, Oxford OX1 3RB, United Kingdom \\ e The Plant Chemetics Laboratory, Max Planck Institute for Plant Breeding Research, Carl-von-Linne Weg 10, 50829 Cologne, Germany
}

\section{A R T I C L E I N F O}

\section{Article history:}

Received 20 November 2015

Received in revised form 8 February 2016

Accepted 11 February 2016

Available online 16 February 2016

\section{Keywords:}

Brassica napus

Nitrogen remobilization efficiency

Soluble proteins

Protease activity

Senescence-associated proteases

Cysteine proteases

\begin{abstract}
A B S T R A C T
Oilseed rape (Brassica napus L.) is a crop plant characterized by a poor nitrogen (N) use efficiency that is mainly due to low $\mathrm{N}$ remobilization efficiency during the sequential leaf senescence of the vegetative stage. As a high leaf $\mathrm{N}$ remobilization efficiency was strongly linked to a high remobilization of proteins during leaf senescence of rapeseed, our objective was to identify senescence-associated protease activities implicated in the protein degradation. To reach this goal, leaf senescence processes and protease activities were investigated in a mature leaf becoming senescent in plants subjected to ample or low nitrate supply. The characterization of protease activities was performed by using in vitro analysis of RuBisCO degradation with or without inhibitors of specific protease classes followed by a protease activity profiling using activity-dependent probes. As expected, the mature leaf became senescent regardless of the nitrate treatment, and nitrate limitation enhanced the senescence processes associated with an enhanced degradation of soluble proteins. The characterization of protease activities revealed that: (i) aspartic proteases and the proteasome were active during senescence regardless of nitrate supply, and (ii) the activities of serine proteases and particularly cysteine proteases (Papain-like Cys proteases and vacuolar processing enzymes) increased when protein remobilization associated with senescence was accelerated by nitrate limitation.

Short statement: Serine and particularly cysteine proteases (both PLCPs and VPEs) seem to play a crucial role in the efficient protein remobilization when leaf senescence of oilseed rape was accelerated by nitrate limitation.
\end{abstract}

(C) 2016 Elsevier Ireland Ltd. All rights reserved.

\footnotetext{
Abbreviations: AP, aspartic protease; $\mathrm{CCV}$, chloroplast vesiculation-containing vesicle; CP, cysteine protease; CXE, carboxylesterase; HN, high nitrate; LN, low nitrate; MES, methylesterase; N, nitrogen; NPC, no probe control; NRE, nitrogen remobilization efficiency; NUE, nitrogen use efficiency; PLCP, papainlike cysteine protease; POPL, prolyloligopeptidase-like protease; RuBisCO (RBC) ribulose-1,5-biphosphate carboxylase/oxygenase; RBCL, large subunit of RuBisCO SAP, senescence associated protease; SAV, senescence associated vesicles; SCPL, serine carboxypeptidase-like protein; VPE, vacuolar processing enzyme.

* Corresponding author at: Université de Caen Normandie, F-14032 Caen, France. Fax: +33231565360.

E-mail addresses: marine.poret@unicaen.fr (M. Poret) balakumaran.chandrasekar@plants.ox.ac.uk (B. Chandrasekar), renier.vanderhoorn@plants.ox.ac.uk (R.A.L.van der Hoorn), jean-christophe.avice@unicaen.fr (J.-C. Avice).
}

\section{Introduction}

Oilseed rape (Brassica napus L.) is the third largest oleaginous crop worldwide and the dominant oilseed crop in northern Europe. It is cultivated for its seeds, from which the extracted oil is used for human food and non-food uses (biofuel, detergents and lubricants) and the cake leftover from processing, which is rich in proteins and micronutrients, is used for animal feed. However, oilseed rape needs a large amount of nitrogen $(\mathrm{N})$ fertilizers (160-250 kg N ha ${ }^{-1}$ year $^{-1}$ ) for its development [1], which can lead to economic losses as well as negative impacts on the environment, and so $\mathrm{N}$ fertilization represents the main operational cost for farmers. That is why, in a context of sustainable agriculture, a reduction in $\mathrm{N}$ inputs combined with the optimization of oilseed rape Nitrogen Use Efficiency (NUE) has become essential [2,3]. Oilseed rape is 

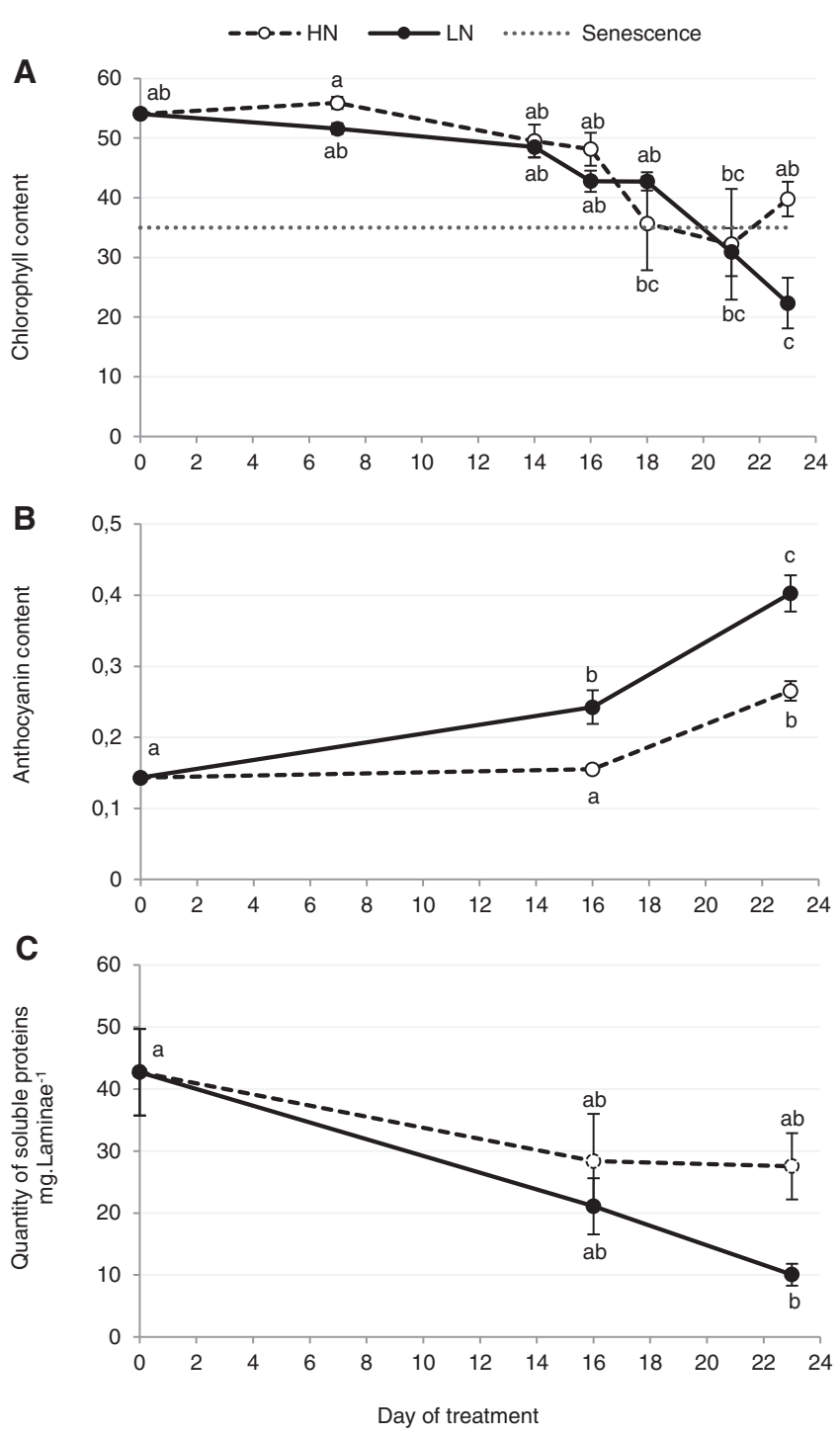

Fig. 1. Changes in parameters associated with senescence in a mature leaf of B. napus L. supplied with ample (HN) or low (LN) nitrate for 23 days. Six-week-old plants were subjected to ample ( $\mathrm{HN}$ : $3.75 \mathrm{mM} \mathrm{NO}_{3}{ }^{-}$) or low nitrogen supply (LN: $0.375 \mathrm{mM}$ $\mathrm{NO}_{3}{ }^{-}$) for 23 days. A mature leaf that became senescent during the experiment (Leaf rank no. 12) was chosen on the basis of its leaf area determined with a LI-COR 300 area metre. Chlorophyll content was measured with a SPAD meter (A). A leaf was considered as senescent when the chlorophyll content is decreased by at least $40 \%$ of the initial value (here the senescence threshold was placed at 35 SPAD units). Anthocyanin content was measured with an optical sensor system (Multiplex $\left.{ }^{\circledR}\right)(B)$ and the quantity of soluble proteins was determined after extraction by proteindye staining [42] (C). Vertical bars indicate \pm SE of the mean $(n=3)$. Statistics are represented by letters ( $p<0.05$, ANOVA, Newman-Keuls test).

characterized by a low NUE because only $50 \%$ of the N from fertilizers is finally recovered in the seeds, while a significant proportion of $\mathrm{N}$ inputs is returned to the environment directly or by the fallen leaves [4]. Moreover, it was shown that the low NUE is mainly due to a weak N Remobilization Efficiency (NRE) [5-7]. Indeed, during the vegetative stages of growth the recycling of foliar $\mathrm{N}$ is not optimal during the 'sequential' leaf senescence, which corresponds to a senescence progression along the axis of the plant that affects leaves as they reach maturity and leads to nutrient remobilization from the older leaves to the younger leaves [3].

In plants, senescence corresponds to the final stage of leaf development and is characterized by the transition from assimilation to remobilization of nutrients [8]. This phenomenon contributes to resource management, recycling and nutrient remobilization

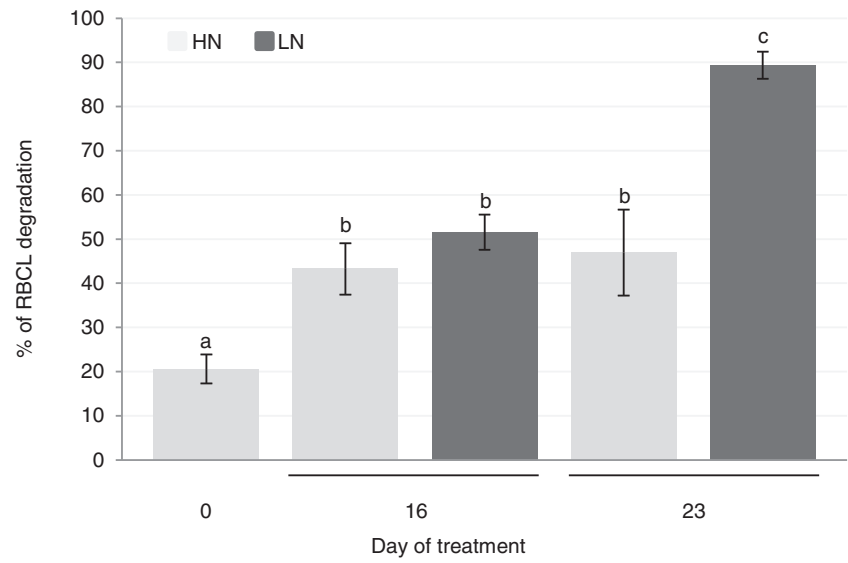

Fig. 2. The degradation of RuBisCO (RBCL) at pH 5.5 increases during leaf senescence of B. napus L. subjected to $\mathrm{N}$ limitation (LN) compared to plants with ample nitrate supply $(\mathrm{HN})$. Soluble proteins were extracted after 0,16 and 23 days of $\mathrm{HN}$ $\left(3.75 \mathrm{mM} \mathrm{NO}_{3}{ }^{-}\right)$or $\mathrm{LN}\left(0.375 \mathrm{mM} \mathrm{NO}_{3}^{-}\right)$treatment and were incubated in sodium acetate buffer $(50 \mathrm{mM}, \mathrm{pH} 5.5)$ for $30 \mathrm{~min}$ at $37^{\circ} \mathrm{C}$. The reaction was stopped by adding ice-cold acetone. To determine the initial quantity of RuBisCO (RBCL, large subunit) another sample was also treated as described above but the reaction was stopped immediately. Samples were separated on SDS-PAGE Stain-free gels (MiniPROTEAN ${ }^{\circledR}$ TGXTM Stain Free), scanned under UV light with a Gel Doc ${ }^{\mathrm{TM}}$ EZ scanner and analysed (for details see Section 2). The degradation of RBCL was calculated as the difference in the quantity between non incubated and incubated samples and expressed as \% of RBCL degradation. Vertical bars indicate \pm SE of the mean $(n=3)$. Statistics are represented by letters ( $p<0.05$, ANOVA, Newman-Keuls test).

efficiency [9] and is essential for plant productivity [10,11]. Leaf senescence, controlled by intrinsic and environmental factors, leads to a sequence of events such as chlorophyll loss, degradation of macromolecules like proteins, dismantling of cellular components, and cell death $[3,12,13]$. Considered as the most important degradation process during leaf senescence, protein breakdown allows the remobilization of $\mathrm{N}$ [14] and the resulting amino acids or peptides are exported to growing parts of the plant via the phloem, leading to an increase in the concentration of amino acids in the phloem sap $[15,16]$.

A recent study has shown that the enhancement of amino acid export and soluble protein degradation in senescing leaves of oilseed rape are crucial for the improvement of $\mathrm{N}$ remobilization [17]. This study on the genotypic variability of foliar N remobilization at the vegetative stage of $B$. napus L. revealed that the export of amino acids is efficient for the ten genotypes studied. Otherwise, the genotype Aviso, unlike other genotypes, is able to maintain its leaf biomass production in response to low nitrate supply and this was essentially due to an improvement of soluble protein degradation. Up to $75 \%$ of leaf $\mathrm{N}$ is located in chloroplasts as proteins, especially in RuBisCO (ribulose-1,5-biphosphate carboxylase/oxygenase, EC 4.1.1.39) and LHCII (Light Harvesting complex II) $[18,19]$ located in the stroma or in the thylakoid membrane, respectively. Moreover, it is known that the RuBisCO degradation during senescence provides much of $\mathrm{N}$ needed to the development of growing organs [20,21]. Accordingly, the improvement of RuBisCO recycling by proteases is crucial for the optimization of $\mathrm{N}$ remobilization [19]. Surprisingly, in fallen leaves of B. napus L., RuBisCO corresponds to one of the major residual proteins [22], suggesting that proteolysis is limiting for $\mathrm{N}$ remobilization during sequential senescence in leaves of oilseed rape.

There are many protease classes involved in protein breakdown during senescence including serine, aspartic, metallo- and cysteine proteases and the proteasome [23]. In wheat, several serine proteases are induced in response to $\mathrm{N}$ starvation during leaf senescence [24]. Moreover, N starvation in oilseed rape plants leads to an increase in an aspartic protease during the first phases of leaf senes- 
A- APs

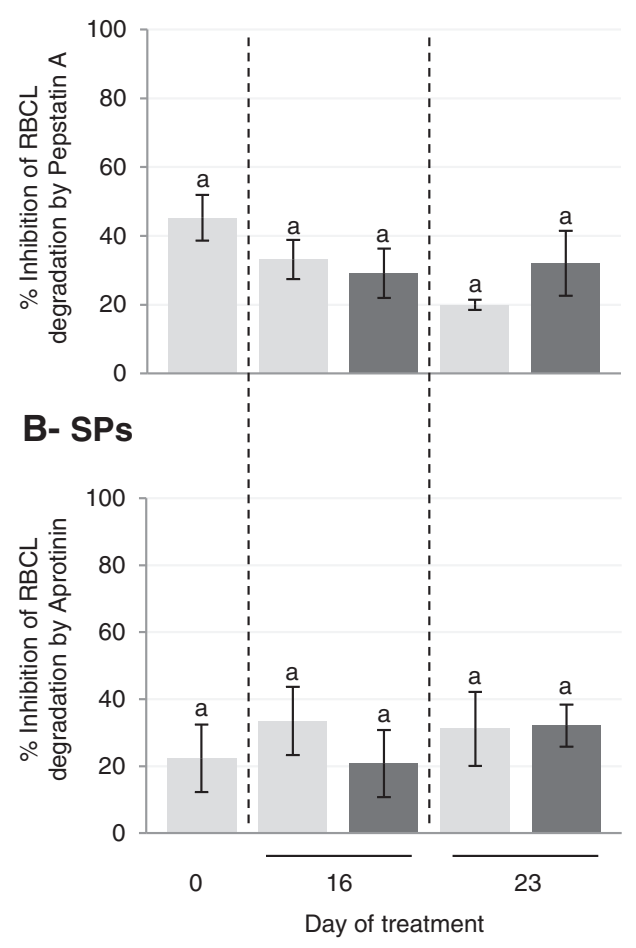

\section{C- PLCPs}

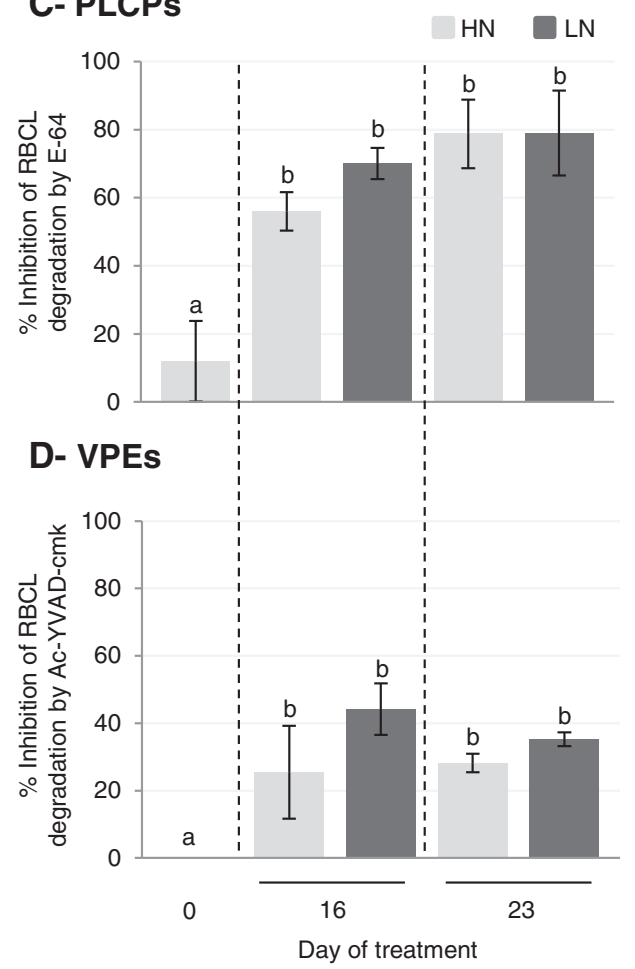

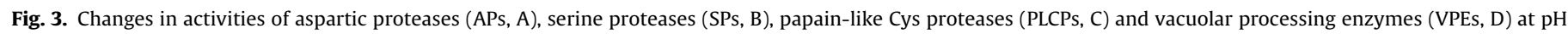

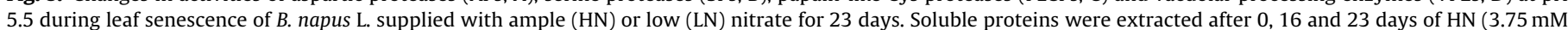

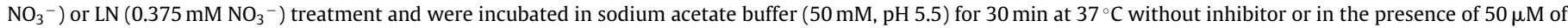

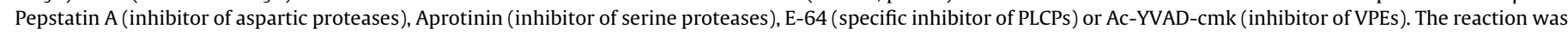

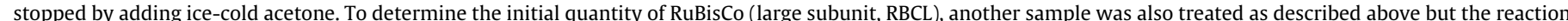

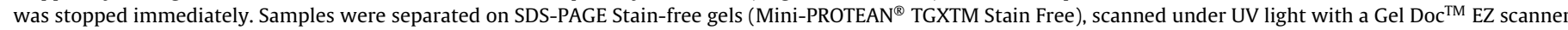

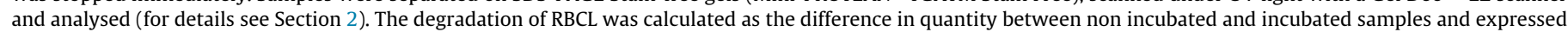

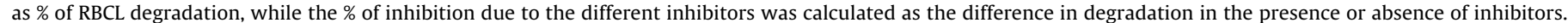
Vertical bars indicate \pm SE of the mean $(n=3)$. Statistics are represented by letters $(p<0.05$, ANOVA, Newman-Keuls test).

cence [25]. Furthermore, aspartic protease CND41 participates in RuBisCO degradation during senescence and CND41 antisense tobacco presented a delay in the senescence process and an accumulation of $\mathrm{N}$ in senescent leaves [26,27]. Metalloproteases have also been implicated in senescence and several genes encoding metalloproteases, particularly FtsH proteases, are induced in senescent leaves of Arabidopsis thaliana [28]. Using proteomics, Desclos et al. [25] showed that nitrate limitation induces a chloroplastic FtsH in senescent leaves of oilseed rape (cv. Capitol). Cysteine proteases might be crucial for the degradation of proteins as they are the most abundant class of proteases up-regulated during leaf senescence-related proteolysis [28,29]. Indeed, in senescent leaves of $A$. thaliana, a high cysteine protease activity was detected in Senescence Associated Vesicles (SAV) [30] and several experiments have demonstrated a role for this class of proteases in RuBisCO degradation [21,31]. Some studies implicated Vacuolar Processing Enzymes (VPEs, a sub-family of cysteine proteases) in leaf senescence. As demonstrated by Sanmartín et al. [32], $\alpha$ VPEs and $\gamma$ VPEs were up-regulated in senescing vegetative organs of $A$. thaliana and encoded proteins might be responsible for the activation of downstream proteases involved in the recycling of amino acids during senescence [33]. Finally, the ubiquitin-proteasome system is also implicated in protein degradation during senescence. The proteasome is able to degrade ubiquitinated, short-lived, regulatory or abnormal proteins. The proteasome consists of the 19S regulatory particle and the $20 \mathrm{~S}$ core protease. The $20 \mathrm{~S}$ core protease contains the catalytic subunits $\beta 1$ (caspase-like activity), $\beta 2$ (trypsin-like activity) and $\beta 5$ (chymotrypsin-like activity) [34]. Proteomic anal- ysis revealed that the catalytic $\beta 1$ subunit was induced during leaf senescence in oilseed rape $[3,25]$.

While RuBisCO degradation during senescence is relatively well studied in other plant species $[19,26,35]$, this process remains largely unknown in oilseed rape. Because soluble protein degradation in senescing leaves of oilseed rape is crucial for the improvement of leaf $\mathrm{N}$ remobilization, the characterization of protease activities is key for comprehension of $\mathrm{N}$ remobilization. Thus, our objective was to identify senescence-associated protease activities implicated in protein remobilization during leaf senescence at the vegetative stage.

As proteases are tightly regulated to prevent damage by uncontrolled proteolytic activities, it is difficult to predict the activity of proteases on the basis of their transcript or protein abundance alone. That is why we focused this work on detecting protease activities by performing an in vitro analysis of the degradation of the RuBisCO large subunit (RBCL), with or without inhibitors of specific protease classes. Additionally, to identify the active proteases associated with the efficient leaf protein degradation during leaf senescence, we used activity-based protein profiling, an original method which allows the detection and identification of proteases in their active states [36].

\section{Material and methods}

\subsection{Chemicals}

E-64, epoxomicin, Ac-YVAD-cmk, diisopropylfluorophosphate (DFP), aprotinin and pepstatin A were from SIGMA-ALDRICH ${ }^{\circledR}$. The 


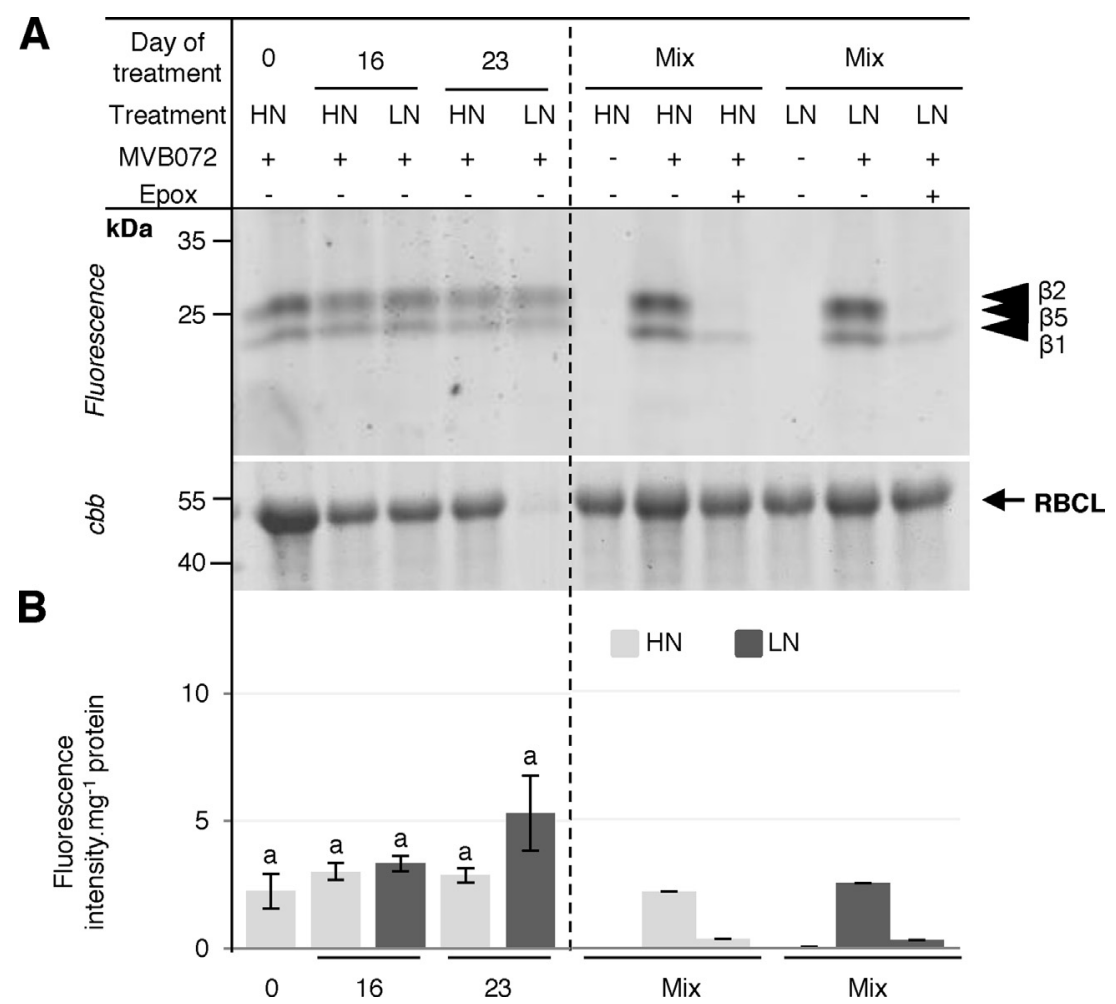

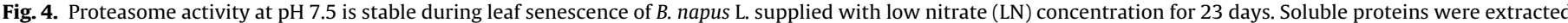

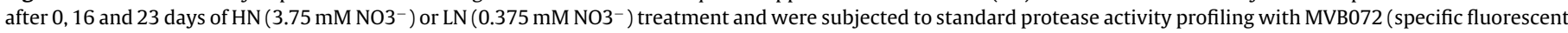

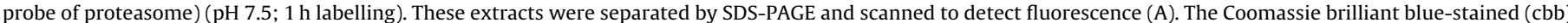

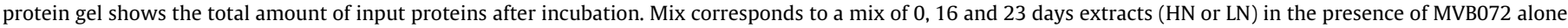

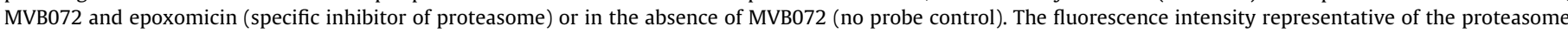

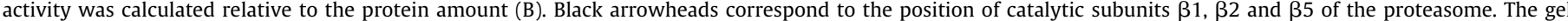

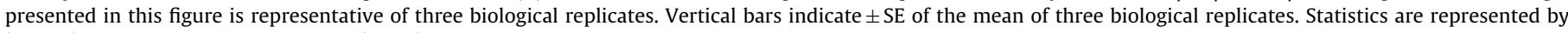
letters $(p<0.05$, ANOVA, Newman-Keuls test).

Table 1

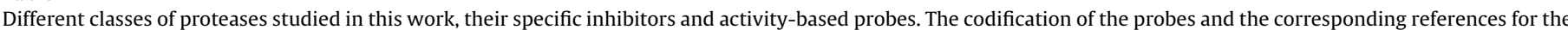
synthesis of these probes are listed.

\begin{tabular}{|c|c|c|c|c|c|}
\hline Class of protease & Sub-family & Inhibitor & Probe with fluorescent tag & Probe with biotin tag & References for the probes \\
\hline \multirow[t]{2}{*}{ Cys proteases } & PLCPs (C1) & E-64 & MV201 & DCG04 & [37] \\
\hline & $\mathrm{VPE}(\mathrm{C} 14)$ & Ac-YVAD-cmk & JOPD1 & - & [38] \\
\hline Ser proteases & Subtilisins (S8), POPLs (S9), SCPLs (S10) & DFP or Aprotinin & $\mathrm{FP}-\mathrm{Rh}$ & FP-biotin & [39] \\
\hline Proteasome & T3 & Epoxomicin & MVB072 & - & [40] \\
\hline Asp proteases & - & Pepstatin A & - & - & - \\
\hline
\end{tabular}

probes MV201, JOPD1, MVB072, FP-Rh, DCG-04 and FP-biotin were available in the laboratory and described in Table 1 [37-40].

\subsection{Plant material and growth conditions}

Oilseed rape (B. napus L. genotype Aviso) plants were cultivated at the vegetative stages in a greenhouse under a $16 \mathrm{~h}$ light regime at $20^{\circ} \mathrm{C}$ (day)/15 ${ }^{\circ} \mathrm{C}$ (night) with a PAR (Photosynthetically Active Radiation) of $400 \mu$ moles photon. $\mathrm{s}^{-1} \mathrm{~m}^{-2}$ at the canopy. After germination, seedlings were transferred into $2.5 \mathrm{~L}$ pots containing mixed vermiculite/perlite $(1: 2 \mathrm{v} / \mathrm{v})$ and cultivated with $25 \%$ Hoagland nutrient solution $(1.25 \mathrm{mM}$ $\mathrm{Ca}\left(\mathrm{NO}_{3}\right)_{2} \cdot 4 \mathrm{H}_{2} \mathrm{O}, 1.25 \mathrm{mM} \mathrm{KNO}_{3}, 0.5 \mathrm{mM} \mathrm{MgSO}_{4}, 0.25 \mathrm{mM} \mathrm{KH}_{2} \mathrm{PO}_{4}$, $0.2 \mathrm{mM}$ EDTA.2NaFe. $3 \mathrm{H}_{2} \mathrm{O}, 14 \mu \mathrm{M} \mathrm{H} \mathrm{H}_{3} \mathrm{BO}_{3}, 5 \mu \mathrm{M} \mathrm{MnSO}_{4}, 3 \mu \mathrm{M}$ $\left.\mathrm{ZnSO}_{4}, 0.7 \mu \mathrm{M}\left(\mathrm{NH}_{4}\right)_{6} \mathrm{Mo}_{7} \mathrm{O}_{24}, 0.7 \mu \mathrm{M} \mathrm{CuSO}_{4}, 0.1 \mathrm{CoCl}_{2}\right)$. After six weeks (Day 0 (D0) of the experiment), plants were supplied with $25 \%$ Hoagland solution containing two different concentrations of nitrate: high (HN: $3.75 \mathrm{mM}$ ) or low nitrate (LN: $0.375 \mathrm{mM} \mathrm{NO}_{3}{ }^{-}$ with a compensation of $\mathrm{Ca}$ and $\mathrm{K}$ elements by adding $1.25 \mathrm{mM}$ $\mathrm{CaCl}_{2} \cdot 2 \mathrm{H}_{2} \mathrm{O}$ and $0.875 \mathrm{mM} \mathrm{KCl}$ ). Leaves were numbered in order of their appearance where leaf rank no. 1 (L1) was the oldest leaf. At D0, a mature leaf becoming senescent during the experiment (leaf rank no. 12 (L12)) was chosen on the basis of its leaf area determined with a LI-COR 300 area meter (LI-COR, Lincoln, NE, USA) and chlorophyll content with a SPAD meter (Soil Plant Analysis Development; Minolta, SPAD-502 model): mean leaf area value of $\left(62.49 \mathrm{~cm}^{2} \pm 9.16 \%\right.$ variation; mean SPAD value of $52.20 \pm 5.52 \%$ variation). Plants were harvested after 0,16 and 23 days of treatment (D0, D16 and D23) and anthocyanin levels of L12 were measured before each harvest by an optical sensor system (Multiplex ${ }^{\circledR}$, Orsay, France) as previously described by D’Hooghe et al. [41]. Laminae of L12 were frozen $\left(-80^{\circ} \mathrm{C}\right)$ and used for proteomics and molecular analyses.

\subsection{Protease activity associated with degradation of RuBisCO $(R B C L)$}

Soluble proteins were extracted by grinding $200 \mathrm{mg}$ of frozen leaf tissue with $500 \mu \mathrm{L}$ citrate-phosphate buffer (20 mM citrate, $160 \mathrm{mM}$ phosphate, $\mathrm{pH} 6.8$ containing $50 \mathrm{mg}$ 


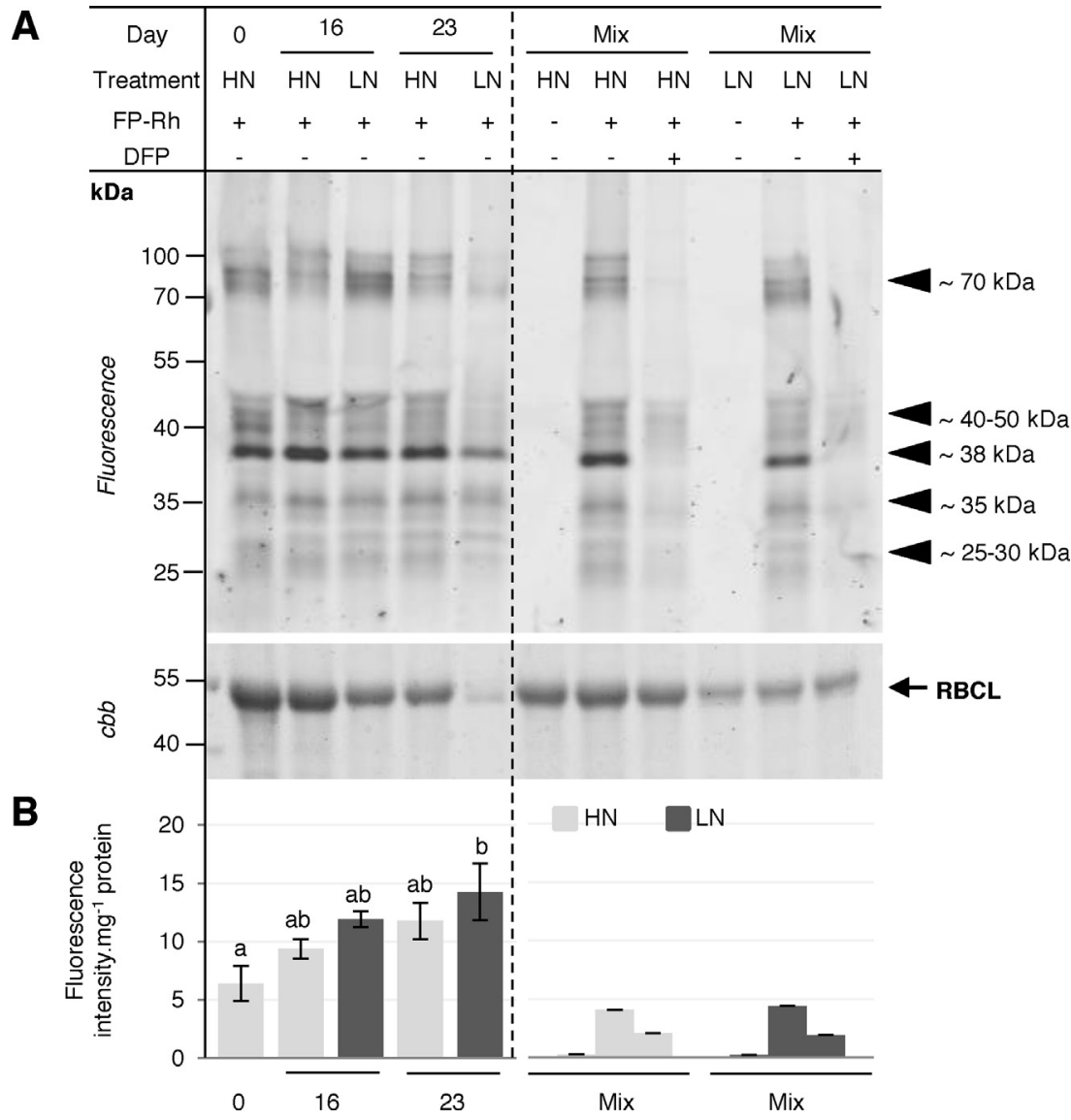

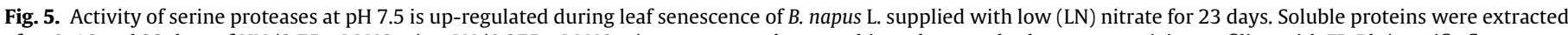

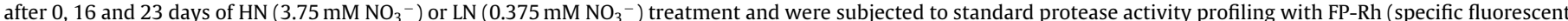

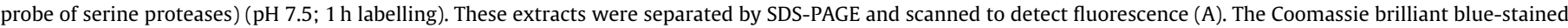

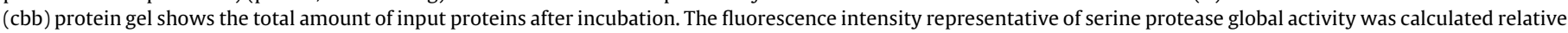

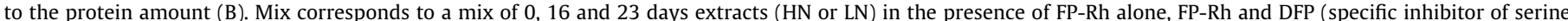

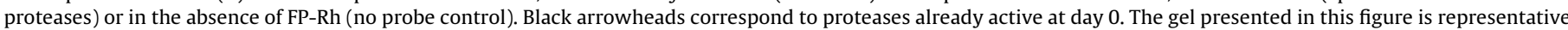

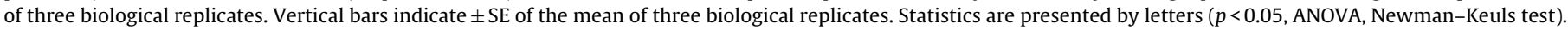

of polyvinylpolypyrrolidone (PVPP)). After centrifugation $(1 \mathrm{~h}$, $12,000 \times g, 4^{\circ} \mathrm{C}$ ), the resulting supernatant containing proteins was used for determination of the quantity of soluble proteins by protein-dye staining [42] using bovine serum albumin (BSA) as standard.

To identify the proteases classes associated with protein remobilization during leaf senescence of plants subjected to nitrate limitation ( $\mathrm{LN})$ or ample nitrate supply $(\mathrm{HN})$, the degradation of RuBisCo large subunit (RBCL) by proteases within the soluble protein extract was studied with or without inhibitors of specific protease classes by using a method modified from Girondé et al. [43] (Supplemental data; Fig. S1). In this method, the RBCL was used as a target of proteolysis because this stromal soluble protein is one of the main substrate of proteases during leaf senescence. Protease activities were determined at pH 5.5 and 7.5. To achieve this, 8 or $12 \mu \mathrm{g}$ of proteins were incubated in a $200 \mu \mathrm{L}$ final volume with sodium acetate buffer $(50 \mathrm{mM}, \mathrm{pH} 5.5)$ in the presence or absence of $50 \mu \mathrm{M}$ of E-64, Ac-YVAD-cmk, aprotinin or pepstatin A (Table 1). Otherwise, to study cysteine proteases, $2 \mathrm{mM}$ of dithiothreitol (DTT) were added in this mix. The incubation was performed for $30 \mathrm{~min}$ at $37^{\circ} \mathrm{C}$ under gentle agitation. Alternatively, 8 or $12 \mu \mathrm{g}$ of proteins were incubated for $90 \mathrm{~min}$ at $37^{\circ} \mathrm{C}$ in a $200 \mu \mathrm{L}$ final volume with Tris-base buffer $(125 \mathrm{mM}$; $\mathrm{pH}$ 7.5) without inhibitor or in the presence of $50 \mu \mathrm{M}$ of epoxomicin. An equal volume of dimethylsulfoxide (DMSO) was added to the No-Inhibitor-Control. Degradation was stopped by adding
$1 \mathrm{~mL}$ of ice-cold acetone. The pellet obtained after centrifugation (15 min, $16,000 \times \mathrm{g}, 4^{\circ} \mathrm{C}$ ) was dissolved in 2X SDS-PAGE gel loading buffer ( $140 \mathrm{mM}$ sodium dodecyl sulfate, $200 \mathrm{mM}$ Tris, $20 \%$ glycerol, $5 \% \beta$-mercaptoethanol, $0.3 \mathrm{mM}$ Bromophenol Blue) and heated at $90^{\circ} \mathrm{C}$ for $10 \mathrm{~min}$. To determine the initial quantity of $\mathrm{RBCL}$, the protein extract was also treated without inhibitor and the proteolytic reaction was stopped immediately by adding $1 \mathrm{~mL}$ of ice-cold acetone, as described above (supplemental Fig. S1). Soluble protein extracts were separated on a $4-15 \%$ gradient in SDS-PAGE precast Stain-free gels (Mini-PROTEAN ${ }^{\circledR}$ TGX $^{\text {TM }}$ Stain Free, Bio-Rad, Marnela-Coquette, France) and scanned under UV light with a Gel Doc ${ }^{\mathrm{TM}}$ EZ scanner (Bio-Rad, Marne-la-Coquette, France). The amount of RBCL (expressed as volume) was quantified by using ImageLab ${ }^{\mathrm{TM}}$ software (Bio-Rad, Marne-la-Coquette, France) according to the manufacturer's instructions. The percentage of RBCL degradation was calculated as the difference in quantity between non incubated and incubated samples without inhibitors. The percentage of inhibition due to the different inhibitors was calculated as the difference in degradation without or in the presence of inhibitors.

\subsection{Protease activity profiling of extracts}

Soluble proteins were extracted by grinding $200 \mathrm{mg}$ of frozen leaf tissue in a microtube and mixing with $1 \mathrm{~mL}$ of water. The extracts were cleared by centrifugation $\left(5 \mathrm{~min}, 13,000 \times \mathrm{g}, 4^{\circ} \mathrm{C}\right.$ ). 
Table 2

LC-MS/MS identification of serine proteases labelled with the probe FP-biotin in a senescent leaves of $B$. napus L. after 23 days of nitrate limitation.

\begin{tabular}{|c|c|c|c|c|c|c|c|c|}
\hline Cutting zone (kDa) & $\begin{array}{l}\text { Protein accession no. } \\
\text { [Brassica napus]/NCBI } \\
\text { accession no. }\end{array}$ & Score & Matches & Match (sig) & Exp. Mass (Da) & Theo. Mass (Da) & $\begin{array}{l}\text { Result of BLAST protein } \\
\text { [Brassica napus]-protein } \\
\text { [organism]/NCBI accession } \\
\text { no./\% identity }\end{array}$ & Class \\
\hline \multirow[t]{5}{*}{70} & Serinepeptidases & & & & & & & \\
\hline & $\begin{array}{l}\text { BnaC05g15670D } \\
\text { [Brassica } \\
\text { napus]/gi|674888482 }\end{array}$ & 91 & 5 & 4 & 82234 & 81657.74 & $\begin{array}{l}\text { PREDICTED: subtilisin-like } \\
\text { protease [Brassica } \\
\text { rapa]/gi|685318103/99\% } \\
\text { Serine-type endopeptidase } \\
\text { SBT5.2 [Arabidopsis } \\
\text { thaliana]/gi|18394832/88\% }\end{array}$ & Subtilisins S8 \\
\hline & $\begin{array}{l}\text { BnaA09g07410D } \\
{[\text { Brassica }} \\
\text { napus]/gi|674914517 }\end{array}$ & 50 & 8 & 4 & 79767 & 79246.24 & $\begin{array}{l}\text { PREDICTED: subtilisin-like } \\
\text { protease [Brassica } \\
\text { rapa]/gi|685354526/85\% } \\
\text { Subtilisin-like protease } \\
\text { [Arabidopsis } \\
\text { thaliana]/gi|18425181/80\% }\end{array}$ & Subtilisins S8 \\
\hline & $\begin{array}{l}\text { BnaCnng55020D } \\
{[\text { Brassica }} \\
\text { napus]/gi|674865926 }\end{array}$ & 41 & 6 & 3 & 79945 & 79310.97 & $\begin{array}{l}\text { PREDICTED: subtilisin-like } \\
\text { protease [Brassica } \\
\text { rapa]/gi|685354526/88\% } \\
\text { Subtilisin-like protease } \\
\text { [Arabidopsis } \\
\text { thaliana]/gi|18425181/78\% }\end{array}$ & Subtilisins S8 \\
\hline & $\begin{array}{l}\text { BnaA03g44620D } \\
\text { [Brassica } \\
\text { napus]/gi|674934691 }\end{array}$ & 37 & 4 & 2 & 145873 & 144766.55 & $\begin{array}{l}\text { PREDICTED: LOW QUALITY } \\
\text { PROTEIN: } \\
\text { tripeptidyl-peptidase 2-like } \\
\text { [Brassica } \\
\text { rapa]/gi|685292411/97\% } \\
\text { Tripeptidyl peptidase II } \\
\text { [Arabidopsis } \\
\text { thaliana]/gi|30685230/92\% }\end{array}$ & Subtilisins S8 \\
\hline \multirow[t]{7}{*}{70} & Serinepeptidases & & & & & & & \\
\hline & $\begin{array}{l}\text { BnaC08g09650D } \\
{[\text { Brassica }} \\
\text { napus]/gi|674896006 }\end{array}$ & 193 & 14 & 6 & 91150 & 90237.22 & $\begin{array}{l}\text { PREDICTED: } \\
\text { acylamino-acid-releasing } \\
\text { enzyme-like isoform X1 } \\
\text { [Brassica } \\
\text { rapa]/gi|685342486/95\% } \\
\text { Acylamino acid-releasing } \\
\text { enzyme [Arabidopsis } \\
\text { thaliana]/gi|42566792/84\% }\end{array}$ & POPLs S9 \\
\hline & $\begin{array}{l}\text { BnaA08g30180D } \\
{[\text { Brassica }} \\
\text { napus]/gi|674878293 }\end{array}$ & 105 & 9 & 4 & 90670 & 89814.83 & $\begin{array}{l}\text { PREDICTED: } \\
\text { acylamino-acid-releasing } \\
\text { enzyme-like isoform X1 } \\
\text { [Brassica } \\
\text { rapa]/gi|685342486/98\% } \\
\text { Acylamino acid-releasing } \\
\text { enzyme [Arabidopsis } \\
\text { thaliana]/gi|42566792/84\% }\end{array}$ & POPLs S9 \\
\hline & $\begin{array}{l}\text { BnaC06g11680D } \\
{[\text { Brassica }} \\
\text { napus]/gi|674926851 }\end{array}$ & 74 & 15 & 8 & 91270 & 90528.61 & $\begin{array}{l}\text { PREDICTED: } \\
\text { acylamino-acid-releasing } \\
\text { enzyme-like isoform X2 } \\
\text { [Brassica } \\
\text { rapa]/gi|685307956/98\% } \\
\text { Acylamino acid-releasing } \\
\text { enzyme [Arabidopsis } \\
\text { thaliana]/gi|42566792/84\% }\end{array}$ & POPLs S9 \\
\hline & $\begin{array}{l}\text { BnaCnng28400D } \\
\text { [Brassica } \\
\text { napus]/gi|674876980 }\end{array}$ & 73 & 13 & 4 & 82654 & 81850.38 & $\begin{array}{l}\text { PREDICTED: prolyl } \\
\text { endopeptidase-like [Brassica } \\
\text { rapa]/gi|685274241/97\% } \\
\text { Prolyl oligopeptidase } \\
\text { [Arabidopsis } \\
\text { thaliana]/gi|79382269/90\% }\end{array}$ & POPLs S9 \\
\hline & $\begin{array}{l}\text { BnaA06g02850D } \\
{[\text { Brassica }} \\
\text { napus]/gi|674910247 }\end{array}$ & 52 & 5 & 1 & 81086 & 80567.57 & $\begin{array}{l}\text { PREDICTED: prolyl } \\
\text { endopeptidase-like [Brassica } \\
\text { rapa]/gi|685314489/99\% } \\
\text { Prolyl oligopeptidase family } \\
\text { protein [Arabidopsis } \\
\text { thaliana]/gi|18403046/92\% }\end{array}$ & POPLs S9 \\
\hline & $\begin{array}{l}\text { BnaA09g04940D } \\
\text { [Brassica } \\
\text { napus]/gi|674913439 }\end{array}$ & 49 & 2 & 2 & 82828 & 82423.77 & $\begin{array}{l}\text { PREDICTED: dipeptidyl } \\
\text { peptidase } 8 \text { [Brassica } \\
\text { rapa]/gi|685260765/99\% } \\
\text { Prolyl oligopeptidase family } \\
\text { protein [Arabidopsis } \\
\text { thaliana]/gi|15237923/87\% }\end{array}$ & POPLs S9 \\
\hline
\end{tabular}


Table 2 (Continued)

\begin{tabular}{|c|c|c|c|c|c|c|c|c|}
\hline Cutting zone (kDa) & $\begin{array}{l}\text { Protein accession no. } \\
\text { [Brassica napus]/NCBI } \\
\text { accession no. }\end{array}$ & Score & Matches & Match (sig) & Exp. Mass (Da) & Theo. Mass (Da) & $\begin{array}{l}\text { Result of BLAST protein } \\
{[\text { Brassica napus]-protein }} \\
\text { [organism]/NCBI accession } \\
\text { no./\% identity }\end{array}$ & Class \\
\hline & $\begin{array}{l}\text { BnaA07g32380D } \\
{[\text { Brassica }} \\
\text { napus]/gi|674946140 }\end{array}$ & 34 & 9 & 1 & 82658 & 81854.18 & $\begin{array}{l}\text { PREDICTED: prolyl } \\
\text { endopeptidase [Brassica } \\
\text { rapa]/gi|685338483/99\% } \\
\text { Prolyl oligopeptidase } \\
\text { [Arabidopsis } \\
\text { thaliana]/gi|79382269/93\% }\end{array}$ & POPLS S9 \\
\hline \multirow[t]{4}{*}{70} & $\begin{array}{l}\text { Other serine } \\
\text { hydrolases }\end{array}$ & & & & & & & \\
\hline & $\begin{array}{l}\text { BnaA06g19010D } \\
\text { [Brassica } \\
\text { napus]/gi|674894935 }\end{array}$ & 64 & 9 & 6 & 35699 & 35550.37 & $\begin{array}{l}\text { PREDICTED: probable } \\
\text { carboxylesterase } 12 \text { [Brassica } \\
\text { rapa]/gi|685320048/99\% } \\
\text { Probable carboxylesterase } 12 \\
\text { [Arabidopsis } \\
\text { thaliana]/gi|15228425/80\% }\end{array}$ & CXE \\
\hline & $\begin{array}{l}\text { BnaC07g46400D } \\
\text { [Brassica } \\
\text { napus]/gi|674961158 }\end{array}$ & 53 & 3 & 3 & 29121 & 31685.13 & $\begin{array}{l}\text { PREDICTED: LOW QUALITY } \\
\text { PROTEIN: methylesterase } \\
\text { 9-like [Brassica } \\
\text { rapa]/gi|685295161/93\% } \\
\text { Methylesterase } 9 \text { [Arabidopsis } \\
\text { thaliana]/gi|15235445/81\% }\end{array}$ & MES \\
\hline & $\begin{array}{l}\text { BnaC01g01910D } \\
\text { [Brassica } \\
\text { napus]/gi|674964800 }\end{array}$ & 35 & 2 & 2 & 28978 & 31540.79 & $\begin{array}{l}\text { PREDICTED: methylesterase } \\
\text { 9-like [Brassica } \\
\text { rapa]/gi|685256421/95\% } \\
\text { Methylesterase } 9 \text { [Arabidopsis } \\
\text { thaliana]/gi|15235445/76\% }\end{array}$ & MES \\
\hline \multirow[t]{5}{*}{$35-40$} & $\begin{array}{l}\text { BnaA06g19010D } \\
\text { [Brassica } \\
\text { napus]/gi|674894935 }\end{array}$ & 56 & 13 & 1 & 35699 & 35550.37 & $\begin{array}{l}\text { PREDICTED: probable } \\
\text { carboxylesterase } 12 \text { [Brassica } \\
\text { rapa]/gi|685320048/ } \\
99 \% \\
\text { Probable carboxylesterase } 12 \\
\text { [Arabidopsis } \\
\text { thaliana]/gi|15228425/80\% }\end{array}$ & CXE \\
\hline & $\begin{array}{l}\text { BnaA02g33270D } \\
\text { [Brassica } \\
\text { napus]/gi|674902269 }\end{array}$ & 52 & 3 & 2 & 17528 & 17424.86 & $\begin{array}{l}\text { PREDICTED: probable } \\
\text { carboxylesterase } 120 \text { [Brassica } \\
\text { rapa]/gi|685278524/ } \\
100 \% \\
\text { Carboxyesterase } 20 \\
{[\text { Arabidopsis }} \\
\text { thaliana]/gi|15241725/63\% }\end{array}$ & CXE \\
\hline & $\begin{array}{l}\text { BnaC07g22410D } \\
\text { [Brassica } \\
\text { napus]/gi|674930271 }\end{array}$ & 44 & 4 & 2 & 38711 & 38449.32 & $\begin{array}{l}\text { PREDICTED: probable } \\
\text { carboxylesterase } 7 \text { isoform X1 } \\
\text { [Brassica } \\
\text { rapa]/gi|685325292/88\% } \\
\text { Probable carboxylesterase } 7 \\
\text { [Arabidopsis } \\
\text { thaliana]/gi|15227669/70\% }\end{array}$ & CXE \\
\hline & $\begin{array}{l}\text { BnaC07g46400D } \\
\text { [Brassica } \\
\text { napus]/gi|674961158 }\end{array}$ & 45 & 3 & 2 & 29121 & 31685.13 & $\begin{array}{l}\text { PREDICTED: LOW QUALITY } \\
\text { PROTEIN: methylesterase } \\
\text { 9-like [Brassica } \\
\text { rapa]/gi|68529516/93\% } \\
\text { Methylesterase } 9 \text { [Arabidopsis } \\
\text { thaliana]/gi|15235445/81\% }\end{array}$ & MES \\
\hline & $\begin{array}{l}\text { BnaA01g00900D } \\
\text { [Brassica } \\
\text { napus]/gi|674958220 }\end{array}$ & 43 & 5 & 2 & 28956 & 28632.94 & $\begin{array}{l}\text { PREDICTED: methylesterase } \\
\text { 9-like [Brassica } \\
\text { rapa]/gi|685256421/99\% } \\
\text { Methylesterase } 9 \text { [Arabidopsis } \\
\text { thaliana]/gi|15235445/77\% }\end{array}$ & MES \\
\hline
\end{tabular}

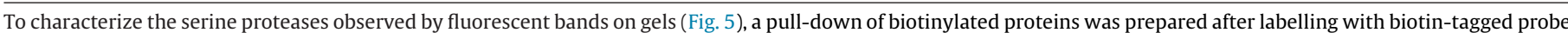

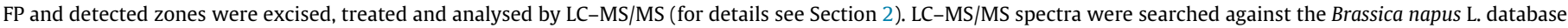

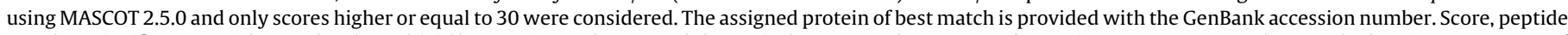

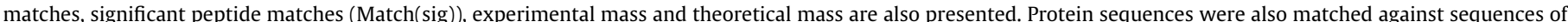

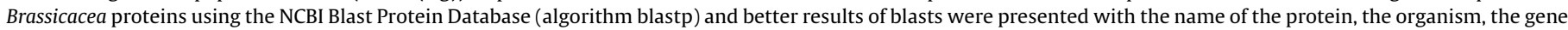
accession number and the percentage of sequence identity. Finally, proteins were classified according to MEROPS database.

The concentration of soluble proteins extract in equivalent serum albumin bovine (BSA) was quantified by protein-dye staining [42].

The in vitro labelling of protease activities was carried out by incubating $20 \mu \mathrm{L}$ of protein extract in a $200 \mu \mathrm{L}$ final volume mix containing $0.5 \mu \mathrm{M}$ of probe (MV201 or JOPD1), $50 \mathrm{mM}$ of NaAc buffer ( $\mathrm{pH} 5.5$ ) and $2 \mathrm{mM}$ DTT. Alternatively, $20 \mu \mathrm{L}$ of protein extract were incubated in a $200 \mu \mathrm{L}$ final volume of $50 \mathrm{mM}$ Tris-base buffer ( $\mathrm{pH} 7.5$ ) containing $0.5 \mu \mathrm{M}$ of MVB072 or $0.25 \mu \mathrm{M}$ of FP-Rh. Samples were incubated for $4 \mathrm{~h}$ (MV201 or JOPD1) or $1 \mathrm{~h}$ (MVB072 or FP-Rh) in the dark under gentle agitation. A mixture of equal volumes of soluble protein extracts of leaves (D0, D16 and D23) treated under $\mathrm{HN}$ or $\mathrm{LN}$ conditions was used as control, and $20 \mu \mathrm{L}$ of each mixture were treated as described above. An equal volume of DMSO was added to the No-Probe-Control (NPC). Moreover, com- 


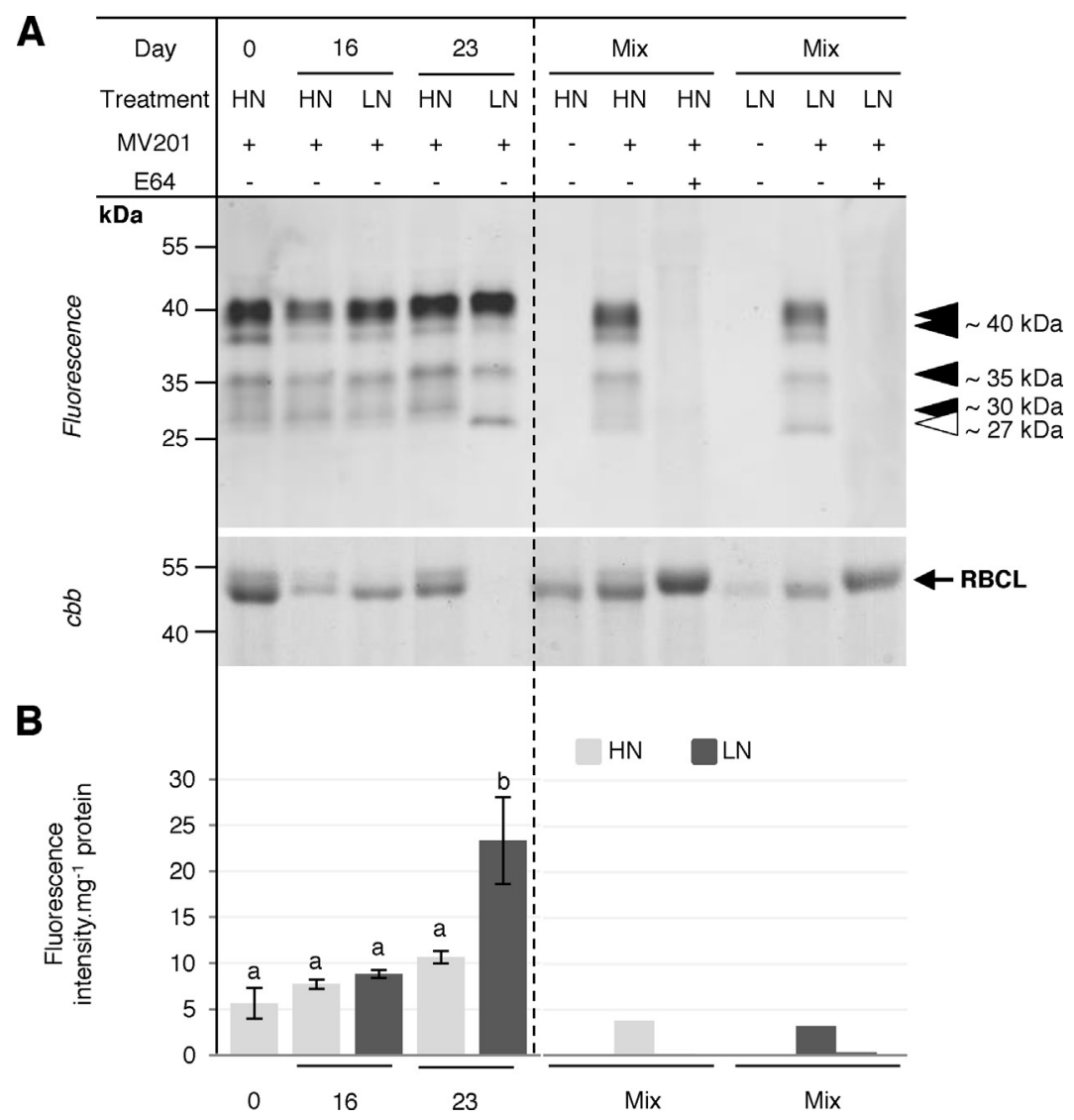

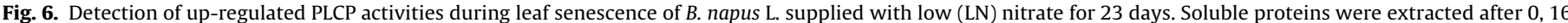

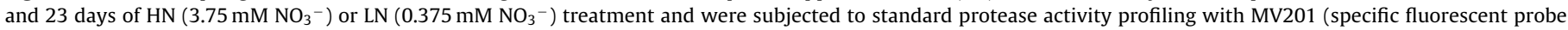

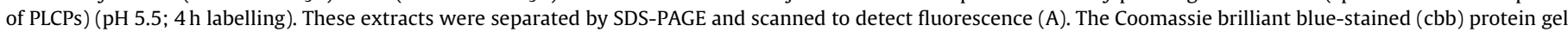

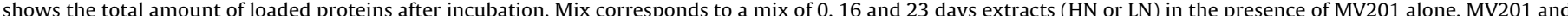

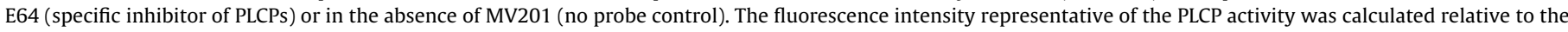

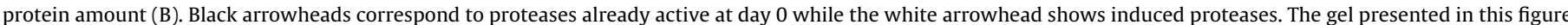

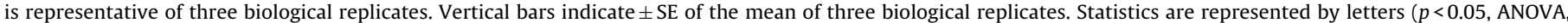
Newman-Keuls test).

petition experiments consisting of a pre-treatment with $50 \mu \mathrm{M}$ of E-64, ac-YVAD-cmk, epoxomicin or DFP for 30 min before adding probes MV201, JOPD1, MVB072 or FP-Rh respectively was carried out in parallel aliquots to control the specificity of probes. Proteins were precipitated after labelling by adding $1 \mathrm{~mL}$ of ice-cold acetone and centrifuging $\left(15 \mathrm{~min}, 16,000 \times \mathrm{g}, 4^{\circ} \mathrm{C}\right)$. The pellet was dissolved in 2X SDS-PAGE gel-loading buffer, heated at $90^{\circ} \mathrm{C}$ for $10 \mathrm{~min}$ and separated on $12 \%$ SDS-PAGE gels. Labelled proteins were visualised by in-gel fluorescence scanning using a Typhoon 9400 scanner (GE Healthcare Life Science) with excitation and emission wavelengths at 532 and $580 \mathrm{~nm}$ respectively. Fluorescence signals were quantified by ImageJ software. Then, gels were stained with Coomassie Brilliant Blue stain (0.5 g CBB G250, 10\% acetic acid, 45\% methanol in ultra-pure water $(\mathrm{v} / \mathrm{v})$ ), destained ( $10 \%$ acetic acid, $40 \%$ methanol in ultra-pure water $(\mathrm{v} / \mathrm{v})$ ) and scanned to control the protein quantity. Fluorescence signals were used to determine specific activity (expressed as fluorescence intensity $\mathrm{mg}^{-1}$ protein).

In order to characterize the proteases observed on gels by the fluorescent bands, a pull-down of biotinylated proteins was performed after labelling with a biotin-tagged probe. For this, $700 \mu \mathrm{g}$ of protein was labelled with $5 \mu \mathrm{M}$ of DCG04 or FP-biotin in labelling buffer ( $50 \mathrm{mM} \mathrm{NaAc}$ pH $5.5,2 \mathrm{mM}$ DTT for DCG04 or $50 \mathrm{mM}$ Tris-buffer, pH 7.5 for FP-biotin). Samples were incubated at room temperature in the dark under gentle agitation for $4 \mathrm{~h}$ (for DCG04) or $1 \mathrm{~h}$ (for FP-biotin). As control, another aliquot of the same sample was treated as described above without probes.
The labelling reaction was stopped and the biotin-proteins were purified using streptavidin beads as described by Chandrasekar et al. [44]. The eluted proteins were separated on $12 \%$ SDS-PAGE gels and the protein gels were stained overnight with SYPRO Ruby (Life Technologies). Proteins were detected by scanning the gel at an excitation wavelength of $460 \mathrm{~nm}$ in ImageQuant LAS 4000 scanner (Filter Y515 Long Pass 500-520 nm; GE Healthcare Life Sciences). Bands were excised and treated with trypsin as described by Kaschani et al. [45]. Then, tryptic peptides were desalted using Stage Tips C8 (Thermo scientific, Bremen, Germany) according to the manufacturer's instructions. Finally, peptide samples were analysed in triplicate by nano-liquid chromatography tandem mass spectrometry (nano-LC-MS/MS) using a Nano-Acquity-UPLC (C18 column of $75 \mu \mathrm{m} \times 250 \mathrm{~mm}, 1.7 \mu \mathrm{m}$ particle size; Waters) coupled to an Orbitrap Elite tandem mass spectrometer (Thermo Scientific) with a resolution of 120,000 full-width half maximum at mass/charge 400, Top 20 precursor ion selection, and fragmentation performed in collision-induced dissociation (CID) mode. The samples were loaded in $99.5 \%$ buffer $\mathrm{A}\left(0.1 \%\right.$ formic acid in $\left.\mathrm{H}_{2} \mathrm{O}\right)$. The gradient used to elute the peptides was started with a 3 min isocratic gradient composed of $3 \%$ buffer $\mathrm{B}\left(0.1 \%\right.$ formic acid in $\left.\mathrm{CH}_{3} \mathrm{CN}\right)$ followed by a linear gradient from $3-40 \%$ of buffer $B$ for 60 min at a flow rate of $250 \mathrm{~nL} \mathrm{~min}^{-1}$ and two washes with $97 \%$ of buffer B for $3 \mathrm{~min}$. The total length of the analysis was $100 \mathrm{~min}$ to allow column re-equilibration. 


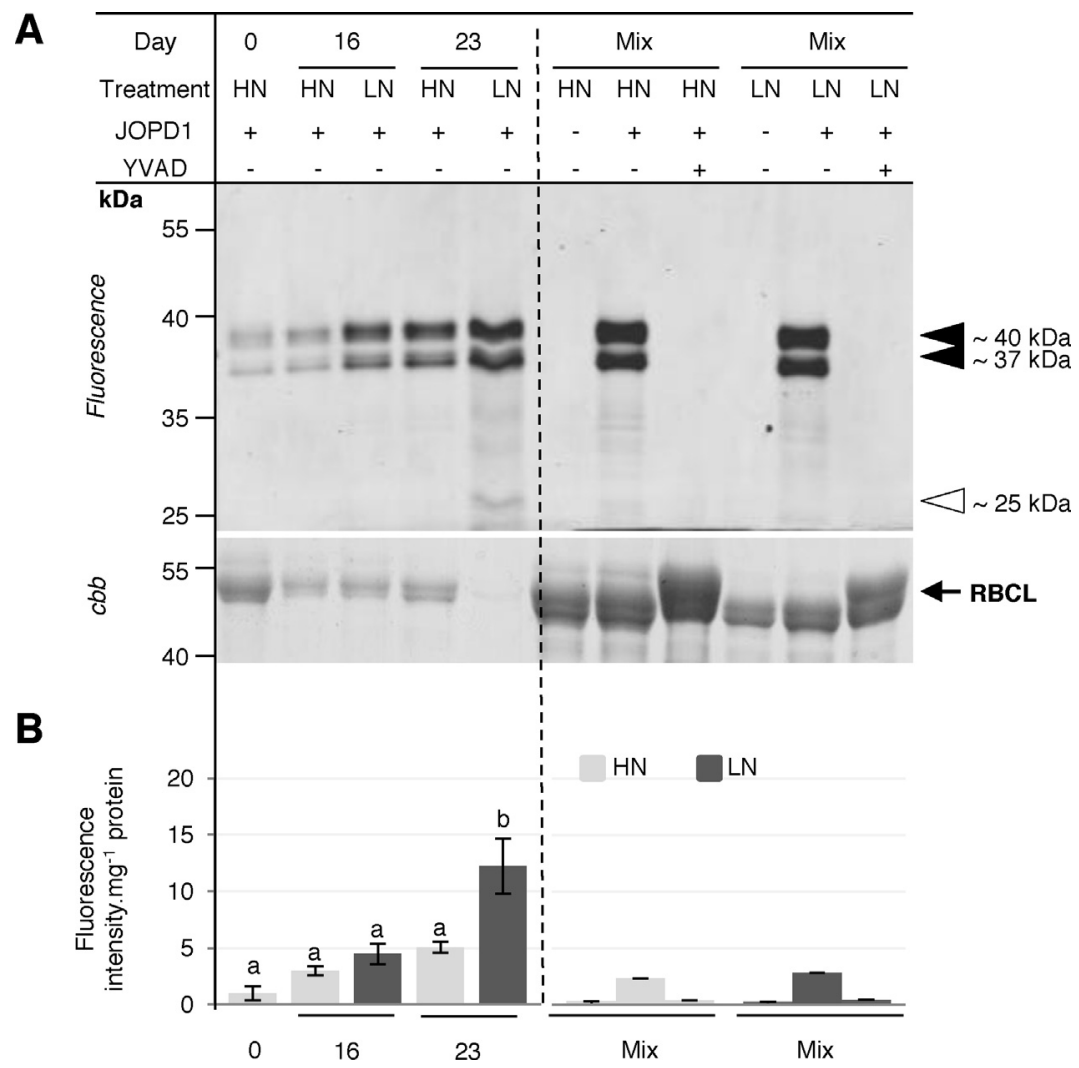

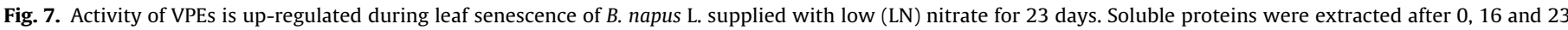

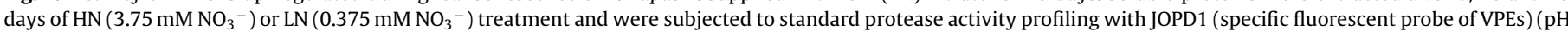

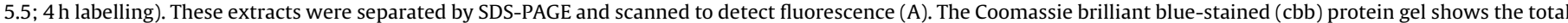

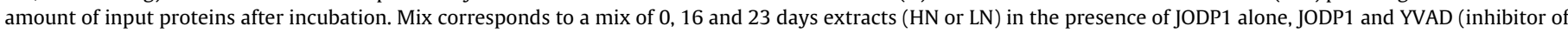

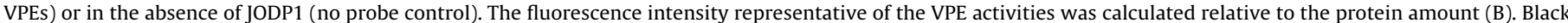

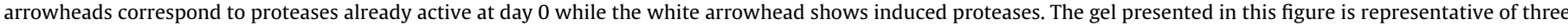
biological replicates. Vertical bars indicate \pm SE of the mean of three biological replicates. Statistics are represented by letters $(p<0.05$, ANOVA, Newman-Keuls test).

Raw MS data were converted into mgf files using MS convert and processed using Mascot software and the following parameters: the error tolerance was fixed at $10 \mathrm{ppm}$ for the precursor ion and at $0.5 \mathrm{Da}$ for the fragment ion. The enzyme used was trypsin and only one missed cleavage was allowed. The post-translational modifications search setting was as follows: fixed modification for cysteine carbamidomethyl and variable modification for asparagine and glutamine deamidation and methionine oxidation. The protein search was performed using the B. napus 20150610 database $(101,040$ sequences; $33,618,840$ residues). The false discovery rate was estimated empirically from decoy hits; identified proteins were filtered to an estimated $1 \%$ FDR (false discovery rate). The assigned protein of best match is provided alongside the GenBank accession number. Score, peptide matches, significant peptide matches (Match(sig)), experimental mass and theoretical mass are also presented. Protein sequences were also matched against sequences of Brassicacea proteins using the NCBI Blast Protein Database (algorithm blastp) and better results of blasts are presented with the name of the protein, the organism, the gene accession number and the percentage of sequence identity. Finally, PLCPs were classified according to the classification of Richau et al. [37] while SPs were classified according to the MEROPS database.

\subsection{Statistical analysis}

For all measurements, three biological repeats were analysed $(n=3)$. All the data are presented as the mean \pm standard error (SE). The normality of the data was studied with the Ryan-Joiner test at $95 \%$. Analysis of variance (ANOVA) and the Newman-Keuls test were used to compare the means by using Microsoft ${ }^{\circledR}$ Excel 2010/XLStat ${ }^{\circledR} 2014$. When the normality law of the data was not respected, the non-parametric test of Kruskal-Wallis was carried out. Statistical significance was postulated at $p<0.05$.

\section{Results and discussion}

In order to characterize key mechanisms that could assist in improving the protein recycling and the leaf NRE of oilseed rape, the objective was to identify senescence-associated proteases (SAPs) implicated in protein degradation during leaf senescence of genotype Aviso, which was previously characterized for its high leaf NRE in response of nitrate limitation [17].

\subsection{Leaf senescence and soluble protein degradation}

To study the changes in physiological and biochemical parameters associated with senescence in a mature leaf of oilseed rape, six week old plants were subjected to ample ( $\mathrm{HN}: 3.75 \mathrm{mM} \mathrm{NO}_{3}{ }^{-}$) or low nitrogen supply ( $\mathrm{LN}: 0.375 \mathrm{mM} \mathrm{NO}_{3}{ }^{-}$) for 23 days. Mature leaves at rank no. 12 (L12) were chosen for the study of senescence progression via the analysis of chlorophyll content, quantity of soluble proteins and protease activities. As expected, a limitation of nitrate (LN) accelerated the leaf senescence processes compared to plants subjected to an ample nitrate supply (HN). Indeed, chlorophyll content was significantly lower after 23 days under LN conditions (22.3 \pm 4 SPAD units) compared to HN supply $(39.8 \pm 3)$ (Fig. 1A). Because leaf redness through anthocyanin accumulation is commonly considered as a symptom of abiotic stresses 
A

B

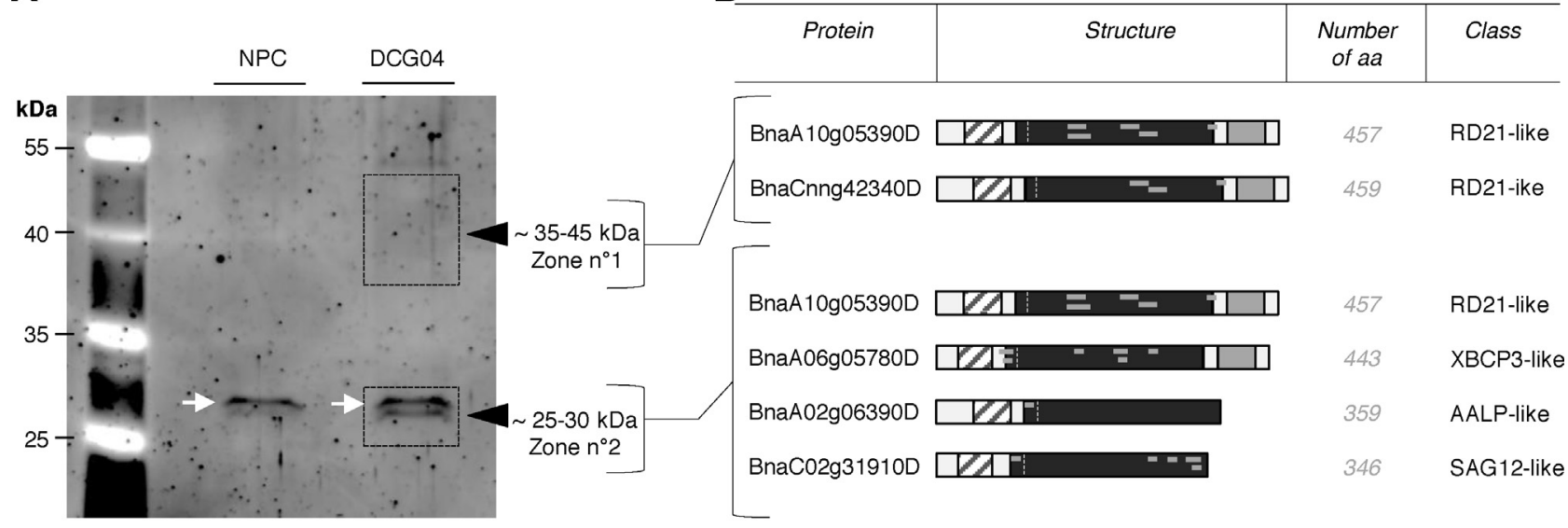

ZLathepsin propeptide inhibitor domain $129 \quad \square$
\begin{tabular}{ll|l}
\hline Peptidase C1A (protease domain) & & ID-ed peptides \\
$\square$ & Granulin domain
\end{tabular}

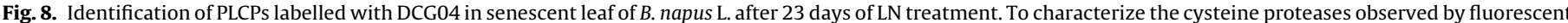

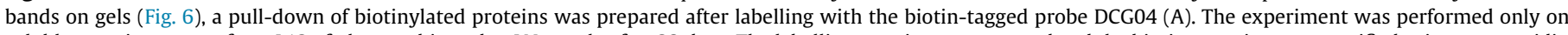

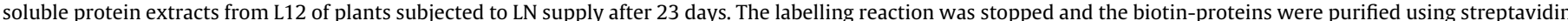

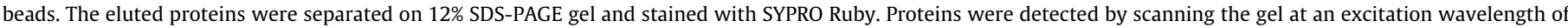

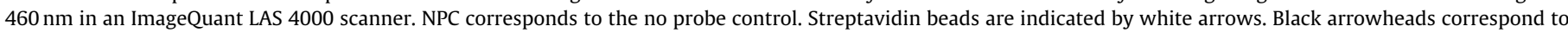

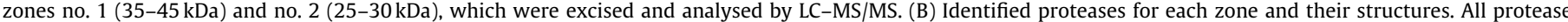

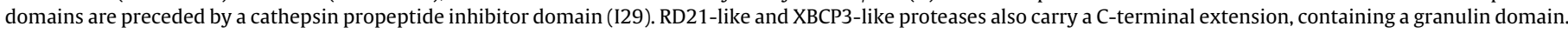

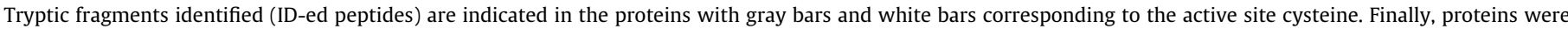
classified according to the classification of Richau et al. [37].

especially in case of mineral limitation such as sulfur, phosphorus and N deficiency in many plants [46-50] including oilseed rape [41], we have studied the changes of anthocyanins in the mature leaf of plants subjected to ample or limited nitrate supply. The level of anthocyanins in L12 had tripled during the 23 days of LN treatment but only doubled under HN treatment (Fig. 1B). These results confirm that $\mathrm{N}$ limitation can cause a rapid induction of anthocyanins in senescing leaves of oilseed rape. Acting as a "sunscreen" in case of excess light and as scavengers for reactive oxidative species, an accumulation of anthocyanins in leaves may protect the photosynthetic apparatus from photodamage and facilitate the recovery of nutrients, especially $\mathrm{N}$, from senescing leaves of oilseed rape as previously reported in other plants [51,52,46]. Furthermore, after 23 days, the nitrate limitation induced the strong degradation of soluble proteins in L12 (-76\%) versus only $-35 \%$ for $\mathrm{L} 12$ of HN plants (Fig. 1C), as described previously for the genotype Aviso [17].

As the N need of growing organs is mainly fulfilled by the degradation of RuBisCO during leaf senescence [20,21], we studied, in particular, the degradation of RBCL (Large subunit of RuBisCO) in vitro at $\mathrm{pH} 5.5$ or 7.5. At $\mathrm{pH} 5.5$ (Fig. 2), the degradation of RBCL by proteases present in extracts of L12 increased at D16 and D23 compared to D0 regardless of the treatment. Nevertheless, after 23 days of nitrate limitation, the RBCL was more degraded $(-89.4 \pm 3.4 \%)$ than under HN supply $(-46.9 \pm 9 \%)$. Otherwise, no degradation of RBCL was observed at $\mathrm{pH} 7.5$ regardless of the date or treatment (data not shown). These results suggest that RBCL degradation in oilseed rape might occur outside the plastid in cellular compartments with an acidic $\mathrm{pH}$ such as SAVs (Senescence Associated Vesicles) or in the central vacuole through the transfer by RCBs (RuBisCO containing bodies) [53-57]. Other studies have shown that chloroplast proteins such as RuBisCO can be partially degraded in the chloroplast itself by chloroplastic proteases $[19,58,59]$. Even if chloroplasts are unable to carry out complete breakdown of the RBCL, it has been reported that a $44 \mathrm{kDa}$ fragment from cleaved RBCL accumulates in chloroplasts isolated from senescing wheat leaves [55,58]. Other chloroplastic proteins such as LHCII can be completely degraded in the plastid at $\mathrm{pH} 7.5[55,60]$, and novel chloroplast vesiculation-containing vesicles (CCVs) have been identified and implicated in chloroplast degradation in Arabidopsis [61]. CCVs containing chloroplast proteins were released from chloroplasts and mobilized to the vacuole for degradation through a pathway that is independent of autophagy and SAVs.

\subsection{Senescence-associated protease (SAP) activities in oilseed rape leaves}

To determine which protease classes could be involved in the degradation of soluble proteins during senescence (Figs. $1 \mathrm{C}$ and 2 ), we studied protease activities in vitro at acidic $\mathrm{pH}(5.5)$ or neutral $\mathrm{pH}$ (7.5) during leaf senescence of plants subjected to $\mathrm{N}$ limitation (LN) compared to plants with ample nitrate supply by two different methods. The first method consisted in studying the RBCL degradation only at acidic $\mathrm{pH}$ (analysis of RBCL degradation was not performed at neutral $\mathrm{pH}$ because of the low contribution of proteolytic activities at this $\mathrm{pH}$, see Section 3.1) in the presence or absence of known inhibitors of specific protease classes. Then, in order to identify active proteases at $\mathrm{pH} 5.5$ and $\mathrm{pH} 7.5$, we performed in parallel a protease activity profiling during leaf senescence using activity-based probes specific for different protease classes [36]. Results indicated that many classes of proteases were active during leaf senescence of oilseed rape, including the proteasome, aspartic, serine and cysteine proteases.

\subsubsection{Aspartic proteases (APs)}

At $\mathrm{pH}$ 5.5, the presence of pepstatin A (inhibitor of aspartic proteases) at D0 inhibited RBCL degradation by $45.2 \pm 6 \%$ at D0 but this was not increased at D16 or D23 under the HN or LN treatments (Fig. 3A). These results showed that APs seem to be active at $\mathrm{pH} 5.5$ during senescence but these activities are not affected by an acceleration of senescence in LN plants. This is in agreement 
Table 3

LC-MS/MS identification of cysteine proteases labelled with the probe DCG04 in senescent leaf of B. napus L. after 23 days of nitrate limitation.

\begin{tabular}{|c|c|c|c|c|c|c|c|c|}
\hline Cutting zone ( $\mathrm{kDa})$ & $\begin{array}{l}\text { Protein accession no. } \\
\text { [Brassica napus]/NCBI } \\
\text { accession no. }\end{array}$ & Score & Matches & Match (sig) & Exp. Mass (Da) & Theo. Mass (Da) & $\begin{array}{l}\text { Result of BLAST protein [Brassica } \\
\text { napus]-protein [organism]/NCBI } \\
\text { accession no./\% identity }\end{array}$ & Classification ofRichau et al. [37] \\
\hline \multirow[t]{2}{*}{$35-45$} & $\begin{array}{l}\text { BnaA10g05390D } \\
{[\text { Brassica }} \\
\text { napus]/gi|674926680 }\end{array}$ & 201 & 14 & 9 & 51650 & 50484.76 & $\begin{array}{l}\text { Senescence-associated cysteine } \\
\text { protease [Brassica } \\
\text { oleracea]/gi|18141285/ } \\
92 \% \\
\text { Cysteine proteinase RD21a [Arabidopsis } \\
\text { thaliana]/gi|18401614/ } \\
83 \%\end{array}$ & RD21A-like \\
\hline & $\begin{array}{l}\text { BnaCnng42340D } \\
{[\text { Brassica }} \\
\text { napus]/gi|674871100 }\end{array}$ & 65 & 5 & 4 & 51828 & 50662.93 & $\begin{array}{l}\text { Senescence-associated cysteine } \\
\text { protease [Brassica } \\
\text { oleracea]/gi|18141285/ } \\
86 \% \\
\text { Cysteine proteinase RD21a [Arabidopsis } \\
\text { thaliana]/gi|18401614/ } \\
82 \%\end{array}$ & RD21A-like \\
\hline \multirow[t]{4}{*}{$25-30$} & $\begin{array}{l}\text { BnaA10g05390D } \\
\text { [Brassica } \\
\text { napus]/gi|674926680 }\end{array}$ & 60 & 5 & 4 & 51650 & 50484.76 & $\begin{array}{l}\text { Senescence-associated cysteine } \\
\text { protease [Brassica } \\
\text { oleracea]/gi|18141285/ } \\
92 \% \\
\text { Cysteine proteinase RD21a [Arabidopsis } \\
\text { thaliana]/gi|18401614/ } \\
83 \%\end{array}$ & RD21A-like \\
\hline & $\begin{array}{l}\text { BnaA06g05780D } \\
{[\text { Brassica }} \\
\text { napus]/gi|674939790 }\end{array}$ & 52 & 8 & 4 & 50101 & 48821.05 & $\begin{array}{l}\text { PREDICTED: low-temperature-induced } \\
\text { cysteine proteinase [Brassica } \\
\text { rapa]/gi|685315355/ } \\
\text { 99\% } \\
\text { Papain-like cysteine peptidase XBCP3 } \\
\text { [Arabidopsis } \\
\text { thaliana]/gi|614600257/89\% }\end{array}$ & ХВСР3-like \\
\hline & $\begin{array}{l}\text { BnaA02g06390D } \\
\text { [Brassica } \\
\text { napus]/gi|674924155 }\end{array}$ & 40 & 2 & 1 & 39682 & 39193.26 & $\begin{array}{l}\text { PREDICTED: thiol protease } \\
\text { aleurain-like [Brassica } \\
\text { rapa]/gi|685271156/ } \\
\text { 96\% } \\
\text { Senescence-associated cysteine } \\
\text { protease [Brassica } \\
\text { oleracea]/gi|18141289/ } \\
\text { 90\% } \\
\text { Thiol protease aleurain [Arabidopsis } \\
\text { thaliana]/gi|18424347| } \\
\text { 89\% }\end{array}$ & AALP-like \\
\hline & $\begin{array}{l}\text { BnaC02g31910D } \\
{[\text { Brassica }} \\
\text { napus]/gi|674945739 }\end{array}$ & 38 & 7 & 1 & 38506 & 38079.10 & $\begin{array}{l}\text { Cysteine protease SAG12 [Arabidopsis } \\
\text { thaliana]/gi|18422605/ } \\
86 \%\end{array}$ & SAG12-like \\
\hline
\end{tabular}

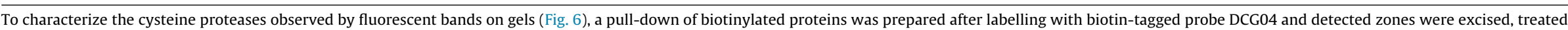

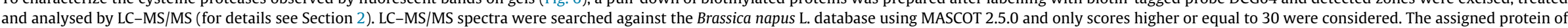

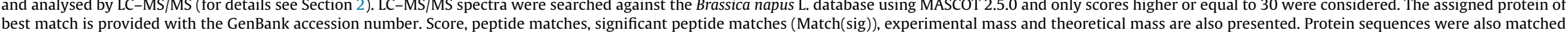

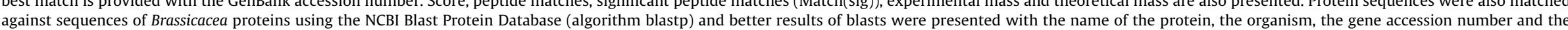
percentage of sequence identity. Finally, proteins were classified according to the classification of Richau et al. [37]. 
with previous proteomic studies reporting that the abundance of an aspartic protease was maintained to a high level during leaf senescence of B. napus [25]. Several studies have demonstrated that the aspartic protease CND41 may play a crucial role during leaf senescence $[14,26,27]$. For instance, in tobacco leaves, CND41 has a high activity at acidic $\mathrm{pH}$ and this protease was able to degrade RBCL in vitro [26]. The present study supports a role for APs during leaf senescence in oilseed rape, but this might be underestimated because pepstatin A does not inhibit tobacco CND41 [26].

\subsubsection{The proteasome}

Because the proteasome is physiologically active under neutral $\mathrm{pH}$, the proteasome activity was studied at $\mathrm{pH}$ 7.5. In our experiment, RBCL was not degraded at $\mathrm{pH} 7.5$ (data not shown) indicating that, at neutral $\mathrm{pH}$, the proteasome is not implicated in the degradation of RBCL. To detect the active proteasome during senescence, an activity-dependent labelling using MVB072 (a specific probe of the proteasome) was performed (Fig. 4, [40]). Labelling with MVB072 allowed the detection of the three catalytic subunits of proteasome: $\beta 1, \beta 2$ and $\beta 5$ (Fig. 4A). Quantification of the sum of activities of these subunits demonstrated that global proteasome activity remained stable during leaf senescence in oilseed rape supplied with ample or low nitrate (Fig. 4B). A similar result was found in dark-induced detached senescing leaves of wheat, which have a constant activity of the 20S proteasome [62]. Several studies also suggest that the proteasome remains functional until late stages of senescence [23]. However, a study of proteomic events associated with $\mathrm{N}$ remobilization during leaf senescence of oilseed rape demonstrated that the $\beta 1$ subunit was accumulated upon $\mathrm{N}$ limitation or starvation [25]. These data suggest that the proteasome plays an important role during senescence, not particularly in the remobilization of Rubisco, but for example to limit the production of reactive oxygen species and/or to contribute to the degradation of other damaged proteins.

\subsubsection{Serine proteases (SPs)}

Because SPs are located in the vacuole [63] and in the chloroplast $[23,64]$, SP activities were studied using (i) RBCL as substrate, under acidic $\mathrm{pH}$ with or without the SP inhibitor aprotinin (Fig. 3B) and (ii) a standard protease activity profiling of SPs using FP-Rh, a specific fluorescent probe of serine proteases performed at pH 7.5 (Fig. 5).

The presence of aprotinin (inhibitor of SPs) at D0 inhibited RBCL degradation by $22.4 \%$, but this was not increased at D16 or D23 under the HN or LN treatments (Fig. 3B). It seemed that SPs were physiologically active under acidic $\mathrm{pH}$ and were able to degrade RBCL but their contribution was relatively low when compared to other protease classes (Fig. 3). A study on leaves of naturally senescing wheat also suggested that SPs participate in RBC degradation and $\mathrm{N}$ remobilization [65]. Otherwise, our data suggest that at neutral $\mathrm{pH}$, SPs do not contribute significantly to RBCL proteolysis during leaf senescence of oilseed rape, irrespective of the nitrate supply.

To verify if SPs were active at neutral $\mathrm{pH}$, an activity-dependent labelling using FP-Rh (a specific probe of SPs) was carried out at pH 7.5 (Fig. 5). Many labelled serine hydrolases were detected after labelling with FP-Rh at $\mathrm{pH} 7.5$ (Fig. 5A). Fluorescent bands were detected at $\sim 70, \sim 40-50, \sim 38, \sim 35$ and $\sim 25-30 \mathrm{kDa}$ and these signals were absent in the no-probe controls and suppressed upon pre-treatment with DFP. Quantification demonstrated that global labelling of serine hydrolases was up-regulated during leaf senescence in response to a limitation of nitrate (Fig. 5B). This was due to an increase in serine hydrolase labelling between D0 and $\mathrm{D} 23$ at $\sim 38-50 \mathrm{kDa}(p=0.007), \sim 35 \mathrm{kDa}(p=0.021)$ and $\sim 25-30 \mathrm{kDa}$ $(p=0.035)$ (data not shown). In response to nitrate limitation, labelling of serine hydrolases at $\sim 70 \mathrm{kDa}$ also increased between D0 and D16.
To identify the labelled SPs, a pull-down of biotinylated proteins from L12 of plants subjected to LN supply over 23 days was carried out after activity-dependent labelling using the biotin-tagged FP probe. Several labelled SPs were identified from signals at 70 and $35-40 \mathrm{kDa}$ (Fig. S2 and Table 2). SPs at $70 \mathrm{kDa}$ were identified as four different subtilisins (S8) and seven prolyloligopeptidase-like proteases (POPLs, S9) (Table 2). In A. thaliana, activity-dependent labelling with similar probes also permitted the identification of POPLs (S9) and subtilase-like (S8) proteases at $70 \mathrm{kDa}[36,66,67,68]$. Some studies have indicated a role for subtilases during natural senescence in wheat and barley leaves [24,63,65]. In addition, during the programmed cell death (PCD) induced by victorin or heat shock treatment, subtilisins (particularly active at neutral $\mathrm{pH}$ ) are required for RuBisCO cleavage [69]. These results may indicate that the RBCL is not degraded at $\mathrm{pH} 7.5$ in vitro. Thus, RBCL does not seem to be the direct target of SPs that are active at neutral $\mathrm{pH}$, in contrast to those active at acidic $\mathrm{pH}$. Interestingly, BnaA03g44620D corresponds to tripeptidyl peptidase II (TPPII), which degrades peptides released by the proteasome [70]. In parallel, four carboxylesterases (CXEs) were identified at both 70 and $35-40 \mathrm{kDa}$ while the three methylesterases (MESs) in B. napus correspond to the methylesterase 9 of $A$. thaliana (AtMES-9). MESs can hydrolyze methylsalicylate, methyljasmonate, and methylindoleacetic acid and may play a role in the regulation of these plant hormones $[71,72]$. Additional experiments are required to characterize the targets of these SPs to determine their role in the leaf senescence of oilseed rape.

\subsubsection{Cysteine proteases (CPS): PLCPs and VPEs}

$\mathrm{CP}$ activities were studied under acidic $\mathrm{pH}$ (5.5) because these proteases are located in the vacuole or in SAVs [30,54]. CP activities were studied firstly using RBCL as substrate, with or without E-64 (inhibitor of papain like cysteine proteases (PLCPs)) or Ac-YVADcmk (inhibitor of vacuolar processing enzymes (VPEs)) (Figs. 3C and D). Additionally, a standard protease activity profiling of PLCPs and VPEs using the probes MV201 or JOPD1, specific fluorescent probes of PLCPs and VPEs respectively, were performed at $\mathrm{pH} 5.5$ (Figs. 6 and 7).

The inhibition of RBCL degradation due to E-64 (inhibitor of PLCPs) was significantly more effective at D23 (78.8\% inhibition (HN); 79\% (LN)) than at D0 (11.9\%) (Fig. 3C). The degradation of RBCL was also significantly inhibited by Ac-YVAD-cmk (inhibitor of VPEs) after 16 or 23 days in L12 of HN and LN plants (Fig. 3D). These results show that the increase in RBCL degradation during senescence (Fig. 2) correlates with a significant increase in PLCP and VPE activities at pH 5.5 focused on the RBCL degradation (Figs. $3 \mathrm{C}$ and $\mathrm{D}$ ). Otherwise, this increase was not significantly different between HN and LN samples.

The results obtained by protease activity profiling at $\mathrm{pH} 5.5$ with MV201 (specific fluorescent probe for PLCPs) or JOPD1 (specific fluorescent probe for VPEs) are presented in Figs. 6 and 7, respectively. Many PLCPs were detected after labelling with MV201 at pH 5.5 (Fig. $6 \mathrm{~A}$ ): at $\sim 40, \sim 30-35$ and $\sim 27 \mathrm{kDa}$ and these signals were absent in the no-probe controls and suppressed upon pre-incubation with PLCP inhibitor E-64. In response to nitrate limitation, the total PLCP labelling was significantly up-regulated in L12 of LN plants after 23 days of treatment (Fig. 6B). Interestingly, one additional band at $\sim 27 \mathrm{kDa}$ appeared only after 23 days of nitrate limitation. Labelling with JOPD1 at pH 5.5 displayed active VPEs at $\sim 40, \sim 37$ and $\sim 25 \mathrm{kDa}$ and these signals were absent in the noprobe controls and suppressed upon pre-incubation with the VPE inhibitor, Ac-YVAD-cmk (Fig. 7A). VPE activities were significantly up-regulated in L12 of plants submitted to LN treatment after 23 days (Fig. 7B). Moreover, one additional fluorescent signal appeared at $\sim 25 \mathrm{kDa}$ after 23 days in response to $\mathrm{LN}$ treatment (Fig. 7A). These results reveal that the increased degradation of soluble proteins 
such as RBCL under LN correlates with an increase in several PLCP and VPE activities at pH 5.5 (Figs. 6 and 7).

It has already been shown that CPs are involved in the degradation of stromal proteins, particularly RBC, during senescence in wheat and tobacco leaves [21,31,55]. A study of dark-induced senescing tobacco leaves showed that RBC degradation in isolated SAVs was blocked by E-64 [57]. More specifically, some PLCPs were up-regulated during senescence in sweet potato, soybean and barley leaves [63,73-75]. In addition, transcript and protein levels of PLCP SAG12 are up-regulated during leaf senescence of oilseed rape subjected to a limitation of $\mathrm{N}$ [25]. These data strongly indicate that PLCPs can be involved in proteolysis associated with leaf senescence in oilseed rape.

In order to identify the labelled PLCPs, a pull-down of biotinylated proteins from L12 of plants subjected to LN supply for 23 days was performed after activity-dependent labelling using the biotintagged DCG04 probe and allowed us to identify five PLCPs (Fig. 8 and Table 3) corresponding to the fluorescent band observed at $27 \mathrm{kDa}$ using MV201 labelling (Fig. 6A).

Two of these PLCPs were classified as RD21A-like proteases according to the classification of Richau et al. [37] and were identified as close homologues of RD21A from $A$. thaliana in the active form at $25 \mathrm{kDa}$ (BnaA10g05390D) and in the intermediate active isoform at $40 \mathrm{kDa}$ (BnaA10g05390D and BnaCnng42340D). RD21 is a PLCP expressed in leaves during senescence and its activity increase in senescing leaves of $A$. thaliana [76,77]. These results strongly suggested that several isoform of $B$. napus proteins may correspond to RD21A in A. thaliana. This protease is synthesized as an inactive pro-protease that is cleaved to the mature/active form in the vacuolar acidic environment, and it has been proposed that the pro-protease might be contained inside ER-bodies with a non-optimum $\mathrm{pH}$ that keeps the protease inactive $[76,78]$.

The three other PLPCs were identified as XBCP3-like, AALP-like and SAG12-like proteases according to the classification of Richau et al. [37]. Interestingly, BnA02g06390D (AALP-like protease) is a close homologue of the senescence-associated cysteine protease, BoCP5, an aleurain-like protein in Brassica oleracea (gi|18141289). In broccoli florets the suppression of BoCP5 delays floret senescence [79]. Consequently, we strongly suspect that BnA02g06390D in oilseed rape has the same proteolysis function during leaf senescence as BoCP5 in B. oleracea. Finally, BnaC02g31910D is homologous to SAG12 of A. thaliana. SAG12 is a senescenceassociated marker gene [80] and encodes for the protease SAG12, which was found specifically in the SAVs [30]. Moreover, Desclos et al. [25] showed that in B. napus leaves subjected to a nitrate limitation, SAG12 expression and SAG12 protein abundance are upregulated during senescence. Consequently, these results indicate that PLCPs related to RD21A or SAG12 proteases could play a crucial role in the efficient proteolysis associated with leaf senescence of $B$. napus, especially in response to $\mathrm{N}$ limitation.

In parallel, VPEs are not implicated in the degradation of chloroplastic proteins such as RBC, but they are responsible for the maturation of several vacuolar proteins [81]. In Arabidopsis leaves, it was suggested that $\alpha$ VPEs and $\gamma$ VPEs (specific to the vegetative organs) regulate the activation of proteins in the lytic vacuole [82]. For instance, it was reported that SAG2 and RD21 genes were induced during leaf senescence and correlated with the induction of VPEs [82]. Previous work has also suggested that $\gamma \mathrm{VPE}$ might activate proteases involved in amino acid recycling during the senescence of $A$. thaliana leaves [33]. Because VPEs might be responsible for the maturation/activation of other proteases such as PLCPs, the increase in VPE activities observed in our study (Figs. 3D and 7) could contribute to an increase in PLCP activities (Figs. 3C and 6). However, some PLCPs, such as RD21A in Arabidopsis [83], do not require VPEs for their activation, so their role in senescence remains unclear.

\section{Concluding remarks}

In conclusion, many classes of proteases are implicated in protein degradation during natural senescence or senescence induced by nitrate limitation in $B$. napus leaves at the vegetative stage. Aspartic proteases, serine proteases, the proteasome and cysteine proteases are highly active at different $\mathrm{pH}$ values associated with different cellular compartments. A limitation of nitrate availability, characterized by an acceleration of senescence processes, leads to an increase in proteolytic activities caused by serine proteases and particularly cysteine proteases (both PLCPs and VPEs). As soluble protein degradation in senescing leaves of oilseed rape is crucial for the improvement of $\mathrm{N}$ remobilization efficiency (NRE), characterization of protease activities is a key for the comprehension of leaf $\mathrm{N}$ remobilization. Moreover, the genotypic differences of leaf NRE observed in response to nitrate limitation [17] might be associated with different or contrasted protease activities during leaf senescence. This study also revealed that activity-based protein profiling is a relevant method for future investigations of genotypic variability of leaf proteolysis efficiency and characterization of protease machinery associated with a high leaf NRE in winter oilseed rape.

\section{Author contributions}

Marine Poret and Jean-Christophe Avice contributed to the experimental design and tissue sampling. Marine Poret and Balakumaran Chandrasekar carried out the protease activity profiling using activity-dependent fluorescent probes. Marine Poret performed other biochemical measurements and proteases analyses, statistical analyses, interpretation of data and drafting the article. Marine Poret, Renier van der Hoorn and Jean-Christophe Avice were involved in revising the manuscript for important intellectual content.

\section{Acknowledgements}

This work was funded by the French National Research Agency (ANR-11-BTBR-004 RAPSODYN-Investments for the Future: Optimisation of the RAPeSeed Oil content and Yield under low Nitrogen input) and by a Ph.D. grant to Mrs. Marine Poret from the French Ministry of Research and the PhD Doctoral School (EDNBISE, Ecole Doctorale Normande Biologie Intégrative, Santé, Environnement). Further financial support was provided by ERA-IB project "PRODuCE", the Max Planck Society, COST CM1004 and the University of Oxford. The authors would like to thank Dr. Nathalie Nési, INRA (UMR 1349 Institut de Génétique, Environnement et Protection des Plantes, INRA, Agrocampus Ouest, Université de Rennes) who is the leader of this ANR-program, Dr. Jacques Trouverie and Dr. Philippe Etienne for their help in protein blasts and for their useful comments on this study, The Target Discovery Institute-Mass Spectrometry Laboratory (University of Oxford) for the proteomic analysis, particularly Marie-Laetitia Thezenas for her kind help with the analysis of proteomics results and finally the UMR EVA technical staff for their skillful assistance. The authors also wish to acknowledge Dr Laurence Cantrill for proofreading and English correction.

\section{Appendix A. Supplementary data}

Supplementary data associated with this article can be found, in the online version, at http://dx.doi.org/10.1016/j.plantsci.2016.02. 011. 


\section{References}

[1] G.W. Rathke, O. Christen, W. Diepenbrock, Effects of nitrogen source and rate on productivity and quality of winter oilseed rape (Brassica napus L.) grown in different crop rotations, Field Crops Res. 94 (2005) 103-113.

[2] T. Behrens, W.L. Horst, F. Wiesler, Effect of rate, timing and form of nitrogen application on yield formation and nitrogen balance in oilseed rape production, in: W.J. Horst, et al. (Eds.), Plant Nutrition: Food Security and Sustainability of Agro-Ecosystems through Basic and Applied Research, Kluwer Academic Publishers, Dordrecht, 2001, pp. 800-801.

[3] J.C. Avice, P. Etienne, Leaf senescence and nitrogen remobilization efficiency in oilseed rape (Brassica napus L.), J. Exp. Bot. 65 (2014) 3813-3824.

[4] J.K. Schjoerring, J.G.H. Bock, L. Gammelvind, C.R. Jensen, V.O. Mogensen, Nitrogen incorporation and remobilization in different shoot components of field-grown winter oilseed rape (Brassica napus L.) as affected by rate of nitrogen application and irrigation, Plant Soil 177 (1995) 255-264.

[5] P. Malagoli, P. Laine, L. Rossato, A. Ourry, Dynamics of nitrogen uptake and mobilization in field-grown winter oilseed rape (Brassica napus) from stem extension to harvest. II. An ${ }^{15} \mathrm{~N}$-labelling-based simulation model of $\mathrm{N}$ partitioning between vegetative and reproductive tissues, Ann. Bot. 95 (2005) 1187-1198.

[6] P. Malagoli, P. Laine, L. Rossato, A. Ourry, Dynamics of nitrogen uptake and mobilization in field-grown winter oilseed rape (Brassica napus) from stem extension to harvest: I. Global N flows between vegetative and reproductive tissues in relation to leaf fall and their residual N, Ann. Bot. 95 (2005) 853-861.

[7] J. Gombert, P. Etienne, A. Ourry, F. Le Dily, The expression patterns of SAG12/Cab genes reveal the spatial and temporal progression of leaf senecence in Brassica napus L. with sensitivity to the environment, J. Exp. Bot. 57 (2006) 1949-1956.

[8] C. Masclaux, M.H. Valadier, N. Brugière, J.F. Morot-Gaudry, B. Hirel, Characterization of the sink/source transition in tobacco (Nicotiana tabacum L.) shoots in relation to nitrogen management and leaf senescence, Planta 21 (2000) 510-518,

[9] A. Guiboileau, R. Sormani, C. Meyer, C. Masclaux-Daubresse, Senescence and death of plant organs: nutrient recycling and developmental regulation, C. R. Biol. 333 (2010) 382-391.

[10] C. Noquet, J.C. Avice, L. Rossato, P. Beauclair, M.P. Henry, A. Ourry, Effects of altered source-sink relationships on $\mathrm{N}$ allocation and vegetative storage protein accumulation in Brassica napus L, Plant Sci. 166 (2004) 1007-1018.

[11] P.L. Gregersen, A. Cutelic, L. Boschian, K. Krupinska, Plant senescence and crop productivity, Plant Mol. Biol. 82 (2013) 603-622.

[12] C.H. Jing, H.G. Nam, Leaf senescence in plants: from model plants to crops still so many unknowns, J. Integr. Plant Biol. 54 (2012) 514-515.

[13] K. Krupinska, M. Mulisch, J. Hollmann, K. Tokarz, W. Zschiesche, H. Kage, K. Humbeck, W. Bilger, An alternative strategy of dismantling of the chloroplasts during senescence observed in a high yield variety of barley, Physiol. Plant. 144 (2012) 189-200.

[14] C. Diaz, T. Lemaître, A. Christ, M. Azzopardi, Y. Kato, F. Sato, J.F. Morot-Gaudry, F. Le Dily, C. Masclaux-Daubresse, Nitrogen recycling and remobilization are differentially controlled by leaf senescence and development stage in Arabidopsis under low nitrogen nutrition, Plant Physiol. 147 (2008) 1437-1449.

[15] C. Masclaux-Daubresse, M. Reisdorf-Cren, K. Pageau, M. Lelandais, O. Grandjean, J. Kronenberger, M.H. Valadier, M. Feraud, T. Jouglet, A. Suzuki, Glutamine synthetase-glutamate synthase pathway and glutamate dehydrogenase play distinct roles in the sink-source nitrogen cycle in tobacco, Plant Physiol. 140 (2006) 444-456.

[16] M.B. Herrera-Rodriguez, R. Perez-vincente, J.M. Maldonado, Expression of asparagine synthetase genes in sunflower (Helianthus annuus) under various environmental stresses, Plant Physiol. Biochem. 45 (2007) 33-38.

[17] A. Girondé, M. Poret, P. Etienne, J. Trouverie, A. Bouchereau, F. Le Cahérec, L. Leport, M. Orsel, M.F. Niogret, C. Deleu, J.C. Avice, A profiling approach of the natural variability of foliar $\mathrm{N}$ remobilization at the rosette stage gives clues to understand the limiting processes involved in the low $\mathrm{N}$ use efficiency of winter oilseed rape, J. Exp. Bot. 66 (2015) 2461-2473.

[18] S. Hörtensteiner, U. Feller, Nitrogen metabolism and remobilization during senescence, J. Exp. Bot. 53 (2002) 927-937.

[19] U. Feller, I. Anders, T. Mae, Rubiscolytics: fate of Rubisco after its enzymatic function in a cell is terminated, J. Exp. Bot. 59 (2008) 1615-1624

[20] K. Demirevska-Kepova, R. Hölzer, L. Simova-Stoilova, U. Feller, Heat stress effects on ribulose-1. carboxylase/oxygenase. Rubisco binding protein and Rubisco activase in wheat leaves, Biol. Plant. 49 (2005) 521-525.

[21] M. Thoenen, B. Herrmann, U. Feller, Senescence in wheat leaves: is a cysteine endopeptidase involved in the degradation of the large subunit of Rubisco, Acta Physiol. Plant. 29 (2007) 339-350.

[22] M. Desclos-Théveniau, L. Coquet, T. Jouenne, P. Etienne, Proteomic analysis of residual proteins in blades and petioles of fallen leaves of Brassica napus, Plant Biol. 17 (2014) 408-418.

[23] I.N. Roberts, C. Caputo, M.V. Criado, C. Funk, Senescence-associated proteases in plants, Physiol. Plant. 145 (2012) 130-139.

[24] I.N. Roberts, S. Passeron, A.J. Barneix, The two main endopeptidases present in darkinduced senescent wheat leaves are distinct subtilisin-like proteases, Planta 224 (2006) 1437-1447.

[25] M. Desclos, P. Etienne, L. Coquet, T. Jouenne, J. Bonnefoy, R. Segura, S. Reze, A Ourry, J.C. Avice, A combined ${ }^{15} \mathrm{~N}$ tracing/proteomics study in Brassica napus reveals the chronology of proteomics events associated with $\mathrm{N}$ remobilisation during leaf senescence induced by nitrate limitation or starvation, Proteomics 9 (2009) 3580-3608

[26] Y. Kato, S. Murakami, Y. Yamamoto, H. Chatani, Y. Kondo, T. Nakano, A. Yokota, F. Sato, The DNA-binding protease, CND41, and the degradation of ribulose-1,5-biphosphate carboxylase/oxygenase in senescent leaves of tobacco, Planta 220 (2004) 97-104

[27] Y. Kato, Y. Yamamoto, S. Murakami, F. Sato, Post-translational regulation of CND41 protease activity in senescent tobacco leaves, Planta 222 (2005) 643-651.

[28] Z. Guo, Transcriptome of Arabidopsis leaf senescence, Plant Cell Environ. 27 (2004) 521-549.

[29] R. Bhalerao, J. Keskitalo, R. Erlandsson, H. Björkbacka, S.J. Birve, J. Karlsson, P. Gardeström, P. Gustafsson, J. Lundeberg, S. Jansson, Gene expression in autumn leaves, Plant Physiol. 131 (2003) 430-442.

[30] M. Otegui, Y.S. Noh, D.E. Martinez, M.G.V. Petroff, L.A. Staehelin, R.M. Amasino, J.J. Guiamet, Senescence-associated vacuoles with intense proteolytic activity develop in leaves of Arabidopsis and soybean, Plant J. 41 (2005) 831-844.

[31] A. Prins, P.D.R. van Heerden, E. Olmos, K.J. Kunert, C.H. Foyer, Cysteine proteinases regulate chloroplast protein content and composition in tobacco leaves: a model for dynamic interactions with ribulose-1,5-bisphosphate carboxylase/oxygenase (Rubisco) vesicular bodies, J. Exp. Bot. 59 (2008) 1935-1950.

[32] M. Sanmartín, L. Jaroszewski, N.V. Raikhel, E. Rojo, Caspases. Regulating death since the origin of life, Plant Physiol. 137 (2005) 841-847.

[33] E. Rojo, J. Zouhar, C. Certer, V. Kovaleva, N.V. Raikhel, A unique mechanism for protein processing and degradation in Arabidopsis thaliana, Proc. Natl. Acad. Sci. U. S. A. 100 (2003) 7389-7394.

[34] C. Gu, I. Kolodziejek, J.C. Misas-Villamil, T. Shindo, T. Colby, M. Verdoes, K.H Richau, J. Schmidt, H. Overkleeft, R.A.L. van der Hoorn, Proteasome activity profiling: a simple, robust and versatile method revealing subunit-selective inhibitors and cytoplasmic, defence-induced proteasome activities, Plant J. 62 (2010) 160-170.

[35] Y. Kato, S. Murakami, N. Takeshi, F. Sato, CND41, a chloroplast DNA-binding protease, is involved in Rubisco degradation, Sci. Access 3 (2001) 1-4.

[36] R.A.L. van der Hoorn, M. Kaiser, Probes for activity-based profiling of plant proteases, Physiol. Plant. 145 (2012) 18-27.

[37] K.H. Richau, F. Kaschani, M. Verdoes, T.C. Pansuriya, S. Niessen, K. Stüber, T. Colby, H.S. Overkleeft, M. Bogyo, R.A.L. van der Hoorn, Subclassification and biochemical analysis of plant papain-like cysteine proteases displays subfamily-specific characteristics, Plant Physiol. 158 (2012) 1583-1599.

[38] H. Lu, B. Chandrasekar, J. Oeljeklaus, J.C. Misas-Villamil, Z. Wang, T. Shindo, M. Bogyo, M. Kaiser, R.A.L. van der Hoorn, Subfamily-specific probes for Cys proteases display dynamic protease activities during seed germination, Plant Physiol. 168 (2015) 1462-1475.

[39] M.P. Patricelli, D.K. Giang, L.M. Stamp, J.J. Burbaum, Direct visualization of serine hydrolase activities in complex proteomes using fluorescent active site-directed probes, Proteomics 1 (2001) 1067-1071.

[40] I. Kolodziejek, J.C. Misas-Villamil, F. Kaschani, J. Clerc, C. Gu, D. Krahn, S. Niessen, M. Verdoes, L.I. Willems, H.S. Overkleeft, M. Kaiser, R.A.L. van der Hoorn, Proteasome activity imaging and profiling characterizes bacterial effector Syringolin A, Plant Physiol. 155 (2011) 477-489.

[41] P. D'Hooghe, S. Escamez, J. Trouverie, J.C. Avice, Sulphur limitation provokes physiological and leaf proteome changes in oilseed rape that lead to perturbation of sulphur, carbon and oxidative metabolisms, BMC Plant Biol. 7 (2013) 13-23.

[42] M.M. Bradford, A rapid and sensitive method for the quantitation of microgram quantities of protein utilizing the principle of protein-dye binding, Anal. Biochem. 72 (1976) 248-254.

[43] A. Girondé, P. Etienne, J. Trouverie, A. Bouchereau, F. Le Cahérec, L. Leport, M. Orsel, M.F. Niogret, N. Nesi, C. Deleu, F. Soulay, C. Masclaux-Daubresse, J.C. Avice, The contrasting management of two oilseed rape genotypes reveals the mechanisms of proteolysis associated with leaf $\mathrm{N}$ remobilization and the respective contributions of leaves and stems to $\mathrm{N}$ storage and remobilization during seed filling, BMC Plant Biol. 21 (2015) 15-59.

[44] B. Chandrasekar, T. Colby, A. Emran Khan Emon, J. Jiang, T.N. Hong, J.G. Villamor, A. Harzen, H.S. Overkleeft, R.A.L. van der Hoorn, Broad-range glycosidase activity profiling, Mol. Cell. Proteom. 13 (2014) 2787-2800.

[45] F. Kaschani, C. Gu, R.A.L. van der Hoorn, Activity-based protein profiling of infected plants, Methods Mol. Biol. 835 (2012) 47-59.

[46] L.D. Noodén, J.W. Hillsberg, M.J. Schneider, Induction of leaf senescence in Arabidopsis thaliana by long days through a light-dosage effect, Physiol. Plant. 96 (1996) 491-495.

[47] L. Chalker-Scott, Environmental significance of anthocyanins in plant stress responses, Photochem. Photobiol. 70 (1999) 1-9.

[48] A.J. Stewart, W. Chapman, G.I. Jenkins, I. Graham, T. Martin, A. Crozier, The effet of nitrogen and phosphorus deficiency on flavonol accumulation in plant tissues, Plant Cell Environ. 24 (2001) 1189-1197.

[49] C. Diaz, V. Saliba-Colombani, O. Loudet, P. Belluomo, L. Moreau, F. Daniel-Vedele, J.F. Morot-Gaudry, C. Masclaux-Daubresse, Leaf yellowing and anthocyanin accumulation are two genetically independent strategies in response to nitrogen limitation in Arabidopsis, Plant Cell Physiol. 47 (2006) 74-83.

[50] M. Peng, D. Hudson, A. Schofield, R. Tsao, R. Yang, H. Gu, Y.M. Bi, S.J. Rothstein, Adaptation of Arabidopsis to nitrogen limitation involves induction of anthocyanin synthesis which is controlled by the NLA gene, J. Exp. Bot. 59 (11) (2008) 2933-2944. 
[51] R.M. Smillie, S.E. Hetherington, Photoabatement by anthocyanin shields photosynthetic systems from light stress, Photosynthetica 36 (1999) 451-463.

[52] W.A. Hoch, E.L. Singsaas, B.H. McCown, Resorption protection: anthocyanins facilitate nutrient recovery in autumn by shielding leaves from potentially damaging light levels, Plant Physiol. 133 (2003) 1296-1305.

[53] A. Chiba, H. Ishida, N.K. Nishizawa, A. Makino, T. Mae, Exclusion of ribulose-1,5-bisphosphate carboxylase/oxygenase from chloroplasts by specific bodies in naturally senescing leaves of wheat, Plant Cell Physiol. 44 (2003) 914-921.

[54] D.E. Martínez, C.G. Bartoli, V. Grbic, J.J. Guiamet, Vacuolar cysteine proteases of wheat (Triticum aestivum L.) are common to leaf senescence induced by different factors, J. Exp. Bot. 58 (2007) 1099-1107.

[55] D.E. Martínez, M.L. Costa, J.J. Guiamet, Senescence-associated degradation of chloroplast proteins inside and outside the organelle, Plant Biol. 10 (2008) $15-22$.

[56] S. Wada, H. Ishida, M. Izumi, K. Yoshimoto, Y. Ohsumi, T. Mae, A. Makino, Autophagy plays a role in chloroplast degradation during senescence in individually darkened leaves, Plant Physiol. 149 (2009) 885-893.

[57] C.A. Carrión, M.L. Costa, D.E. Martínez, C. Mohr, K. Humbeck, J.J. Guiamet, In vivo inhibition of cysteine proteases provides evidence for the involvement of 'senescence-associated vacuoles' in chloroplast protein degradation during dark-induced senescence of tobacco leaves, J. Exp. Bot. 64 (2013) 4967-4980.

[58] N. Kokubun, H. Ishida, A. Makino, T. Mae, The degradation of the large subunit of ribulose-1. carboxylase/oxygenase into the 44-kDa fragment in the lysates of chloroplasts incubated in darkness, Plant Cell Physiol. 43 (2002) 1390-1395

[59] L.F. Zhang, Q. Rui, P. Zhang, X.Y. Wang, L.L. Xu, A novel 51-kDa fragment of the large subunit of ribulose-1. carboxylase/oxygenase formed in the stroma of chloroplasts in dark-induced senescing wheat leaves, Physiol. Plant. 131 (2007) 64-71.

[60] A. Zelisko, M. García-Lorenzo, G. Jackowski, S. Jansson, C. Funk, AtFtsH6 is involved in the degradation of the light-harvesting complex II during high-light acclimation and senescence, Proc. Natl. Acad. Sci. U. S. A. 102 (2005) 13699-13704.

[61] S. Wang, E. Blumwald, Stress-induced chloroplast degradation in Arabidopsis is regulated via a process independent of autophagy and senescence-associated vacuoles, Plant Cell 26 (2014) 4875-4888.

[62] I. Roberts, P.F. Murray, S. Passeron, A.J. Barneix, The activity of the 20 S proteasome is maintained in detached wheat leaves during senescence in darkness, Plant Physiol. Biochem. 40 (2002) 161-166.

[63] D.L. Parrott, K. McInnerney, U. Feller, A.M. Fischer, Steam-girdling of barley (Hordeum vulgare) leaves leads to carbohydrate accumulation and accelerated leaf senescence, facilitating transcriptomic analysis of senescence-associated genes, New Phytol. 176 (2007) 56-59.

[64] C.M. Antão, F.X. Malcata, Plant serine proteases: biochemical, physiological and molecular features, Plant Physiol. Biochem. 43 (2005) 637-650.

[65] I.N. Roberts, C. Caputo, M. Kade, M.V. Criado, A. Barneix, Subtilisin-like serine proteases involved in $\mathrm{N}$ remobilization during grain filling in wheat, Acta Physiol. Plant. 33 (2011) 1997-2001.

[66] F. Kaschani, C. Gu, S. Niessen, H. Hoover, B.F. Cravatt, R.A.L. van der Hoorn, Diversity of serine hydrolase activities of unchallenged and botrytis-infected Arabidopsis thaliana, Mol. Cell. Proteom. 8 (2009) 1082-1093.

[67] F. Kaschani, S. Nickel, B. Pandey, B.F. Cravatt, M. Kaiser, R.A.L. van der Hoorn, Selective inhibition of plant serine hydrolases by agrochemicals revealed by competitive ABPP, Bioorg. Med. Chem. 20 (2011) 597-600.

[68] S. Nickel, F. Kaschani, T. Colby, R.A.L. van der Hoorn, M. Kaiser, A para-nitrophenol phosphonate probe labels distinct serine hydrolases of Arabidopsis, Bioorg. Med. Chem. 20 (2011) 601-606.

[69] W.C. Coffeen, T.J. Wolpert, Purification and characterization of serine proteases that exhibit caspase-like activity and are associated with programmed cell death in Avena sativa, Plant Cell 16 (2004) 857-873.

[70] A.J. Book, P. Yang, M. Scalf, L.M. Smith, R.D. Vierstra, Tripeptidyl peptidase II. An oligomeric protease complex from Arabidopsis, Plant Physiol. 138 (2005) 1046-1057.

[71] A.C. Vlot, P.P. Liu, R.K. Cameron, S.W. Park, Y. Yang, D. Kumar, F. Zhou, T. Padukkavidana, C. Gustafsson, E. Picherski, D.F. Klessig, Identification of likely orthologs of tobacco salicylic acid binding protein 2 and their role in systemic acquired resistance in Arabidopsis thaliana, Plant J. 53 (2008) 445-456.

[72] Y. Yang, R. Xu, C. Ma, A.C. Vlot, D.F. Klessig, E. Pichersky, Inactive methyl indole-3-acetic acid ester can be hydrolyzed and activated by several esterases belonging to the AtMES esterase family of Arabidopsis, Plant Physiol. 147 (2008) 1034-1045.

[73] D.L. Parrott, J.M. Martin, A.M. Fischer, Analysis of barley (Hordeum vulgare) leaf senescence and protease gene expression: a family C1A cysteine protease is specifically induced under conditions characterized by high carbohydrate but low to moderate nitrogen levels, New Phytol. 187 (2010) 313-331.

[74] H.J. Chen, C.T. Su, C.H. Lin, G.J. Huang, Y.H. Lin, Expression of sweet potato cysteine protease SPCP2 altered developmental charateristics and stress responses in transgenic Arabidopsis plants, J. Plant Physiol. 167 (2010) $838-847$.

[75] B. Esteban-García, J.A. Garrido-Cardenas, D.L. Alonso, F. García-Maroto, A distinct subfamily of papain-like cystein proteinases regulated by senescence and stresses in Glycine max, J. Plant Physiol. 167 (2010) 1101-1108.

[76] K. Yamada, R. Matsushima, M. Nishimura, I. Hara-Nishimura, A slow maturation of a cysteine protease with a granulin domain in the vacuoles of senescing Arabidopsis leaves, Plant Physiol. 127 (2001) 1626-1634.

[77] R.A.L. van der Hoorn, M.A. Leeuwenburgh, M. Bogyo, M.H.A.J. Joosten, S.C. Peck, Activity profiling of papain-like cysteine proteases in plants, Plant Physiol. 135 (2004) 1170-1178.

[78] K. Müntz, Protein dynamics and proteolysis in plant vacuoles, J. Exp. Bot. 58 (2007) 2391-2407.

[79] J.R. Eason, D.J. Ryan, L.M. Watson, D. Hedderley, M.C. Christey, R.H. Braun, S.A. Coupe, Suppression of the cysteine protease aleurain, delays floret senescence in Brassica oleracea, Plant Mol. Biol. 57 (2005) 645-657.

[80] K.N. Lohman, S. Gan, M.C. John, R.M. Amasino, Molecular analysis of natural leaf senescence in Arabidopsis thaliana, Physiol. Plant. 92 (1994) 322-328.

[81] I. Hara-Nishimura, N. Hatsugai, S. Nakaune, M. Kuroyanagi, M. Nishimura, Vacuolar processing enzyme: an executor of plant cell death, Curr. Opin. Plant Biol. 8 (2005) 404-408

[82] T. Kinoshita, K. Yamada, N. Hiraiwa, M. Kondo, M. Nishimura, I. Hara-Nishimura, Vacuolar processing enzyme is up-regulated in the lytic vacuoles of vegetative tissues during senescence and under various stressed conditions, Plant J. 19 (1999) 43-53.

[83] C. Gu, M. Shabab, R. Strasser, P.J. Wolters, T. Shindo, M. Niemer, F. Kaschani, L. Mach, R.A.L. van der Hoorn, Post-translational regulation and trafficking of the granulin-containing protease RD21 of Arabidopsis thaliana, PLoS One 7 (2012) e32422. 